Article

\title{
Synthesis, Docking Study and $\beta$-Adrenoceptor Activity of Some New Oxime Ether Derivatives
}

\author{
Hazem A. Ghabbour ${ }^{1,2, *}$, Eman R. El-Bendary ${ }^{1}$, Mahmoud B. El-Ashmawy ${ }^{1}$ and \\ Mohamed M. El-Kerdawy ${ }^{1}$
}

1 Department of Medicinal Chemistry, Faculty of Pharmacy, Mansoura University, Mansoura 35516, Egypt

2 Department of Pharmaceutical Chemistry, Faculty of Pharmacy, King Saud University, P. O. Box 2457, Riyadh 11451, Saudi Arabia

* Author to whom correspondence should be addressed; E-Mail: hghabbour@mans.edu.eg or ghabbourh@yahoo.com; Tel.: +9-665-693-52985; Fax: +9-661-4676-220.

Received: 17 February 2014; in revised form: 5 March 2014 / Accepted: 11 March 2014 /

Published: 20 March 2014

\begin{abstract}
A new series of oxime ethers $4 \mathbf{a}-\mathbf{z}$ was designed and synthesized to test the blocking activity against $\beta_{1}$ and $\beta_{2}$-adrenergic receptors. Docking of these ether derivatives into the active site of the identified $3 \mathrm{D}$ structures of $\beta_{1}$ and $\beta_{2}$-adrenergic receptors showed MolDock scores comparable to those of reference compounds. Biological results revealed that the inhibition effects on the heart rate and contractility are less than those of propranolol. Nevertheless, the two compounds $\mathbf{4 p}$ and $\mathbf{4 q}$ that displayed the highest negative MolDock score with $\beta_{2}$-adrenergic receptors showed $\beta_{2}$-antagonistic activity by decreasing salbutamol relaxation of precontracted tracheal strips, which indicates the importance of a chlorothiophene moiety in the hydrophobic region for best complementarity with $\beta_{2}$ receptors. On other hand, the presence of a homoveratryl moiety increases the MolDock score of the tested compounds with the $\beta_{1}$ receptor.
\end{abstract}

Keywords: synthesis; screening; docking; oxime ethers; adrenergic; $\beta$-receptors

\section{Introduction}

$\beta$-Adrenoceptor blockers represent an important class of therapeutics due to their usefulness in a number of diseases, such as angina pectoris, arrhythmia and hypertension. They may also be used in 
migraine, glaucoma, tremors, hyperthyroidism, and anxiety-provoking situations [1]. Based on the chemical structure of the classical aryloxypropanolamine $\beta$-adrenoceptor blockers, Imbs et al. [2] showed that the insertion of an oxime ether in place of the typical ether linkage retained $\beta_{2}$-antagonist activity; e.g., IPS 339 (Figure 1). Other oxime ethers were also synthesized and reported to have $\beta_{2}$-blocking activity with high potency, e.g., falintolol (Figure 1) [3,4]. Although the above mentioned cardiovascular effects are mainly attributed to $\beta_{1}$-blockage, $\beta_{2}$-adrenoceptor antagonists might be potentially interesting as experimental tools [5], or for testing in pulmonary arterial hypertension [6].

Figure 1. Chemical structures of IPS 339 and falintolol.

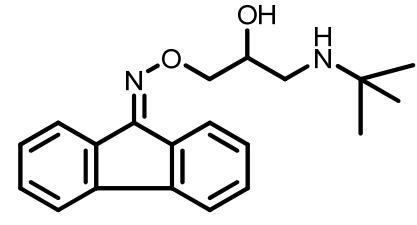

IPS 339

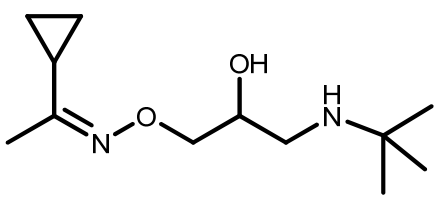

Falintolol

In the present investigation, we intended to further explore the general structure of oxime ethers through different molecular modifications, and to test the $\beta_{1}$ - and $\beta_{2}$-adrenoceptor blocking activity of the new compounds. The manipulation strategy involves usage of aromatic, alicyclic, bicyclic and/or heterocyclic moieties to represent the hydrophobic part of the molecule, in order to verify the importance of these moieties in binding with the receptor, and hence on the selectivity of the resulted compounds. Obeying the SAR of the $\beta$-blockers, the already known $\beta$-directing isopropyl and tert-butyl group as well as the dimethoxyphenylethyl moiety were selected as the best representatives of alkyl and arylalkylamines. The synthesis, molecular docking and preliminary biological evaluation of the thus designed $O$-(3-alkylamino-2-hydroxypropyl)oxime derivatives $\mathbf{4 a -} \mathbf{z}$ are reported herein.

\section{Results and Discussion}

\subsection{Chemistry}

The general method to prepare the final compounds $\mathbf{4 a}-\mathbf{z}$ is outlined in Scheme 1. The oximes $\mathbf{2} \mathbf{a}-\mathbf{j}$ (Table 1), required in the present work, have been obtained via reaction of the corresponding ketones $\mathbf{1 a}-\mathbf{j}$ with hydroxylamine hydrochloride, as previously reported [3,7-14]. The two oxime geometric isomers were not separated, based on the previously reported absence of appreciable difference in $\beta$ - blocking activity between the $E$ - and $Z$-isomers of the final oxime ether derivatives [15]. Accordingly, compounds $\mathbf{2 a - j}$ were reacted with epichlorohydrin, in dry DMF, in the presence of $\mathrm{NaH}$, to afford the epoxy compounds 3a-j (Table 1). In the ${ }^{1} \mathrm{H}-\mathrm{NMR}$ spectra, the oxirane protons appeared mostly as multiplets at $\delta 2.4-3.0 \mathrm{ppm}$, while the $-\mathrm{OCH}_{2}$ - protons appeared as two doublets of doublets at $\delta$ 3.7-4.4 ppm (Experimental part). The target compounds $4 \mathbf{a}-\mathbf{z}$ (Table 2) were prepared by reaction of the epoxy compounds $\mathbf{3 a}-\mathbf{j}$ with excess of isopropylamine, tert-butylamine or homoveratrylamine in toluene, in a sealed tube, at $120^{\circ} \mathrm{C}$. 
Scheme 1. Synthesis of the oxime ethers $4 \mathbf{a}-\mathbf{z}$.

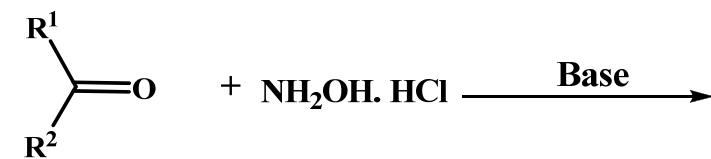

1a-j<smiles>[R]C([R])=NO</smiles>

2a-j

1) $\mathrm{NaH} / \mathrm{DMF}$

2)<smiles>ClCC1CO1</smiles><smiles>[R]C([R])=NOCC1CO1</smiles><smiles>[R1]C(N)=NOCC(O)CN</smiles>

4a-z

Table 1. Oximes $\mathbf{2 a}-\mathbf{j}$ and oxime ethers $\mathbf{3 a}-\mathbf{j}$.<smiles>CC=NO</smiles>

2a-j<smiles>[1H]C=NOCC1CO1</smiles>

Compound No.

\subsection{Molecular Docking}

Molecular docking is a very popular method employed to investigate molecular association. It is particularly useful in the drug discovery arena to study the binding of small molecules (ligands) to macromolecules (receptors or enzymes). Docking-based drug design using structural biology remains 
one of the most logical and esthetically pleasing approaches in the drug discovery process. The structured knowledge of the binding capabilities of the active site residues to specific groups on the agonist or antagonist leads to various proposals for the synthesis of very specific agents with a high probability of biological action [16].

Table 2. $O$-(3-Alkylamino-2-hydroxypropyl)oxime derivatives $\mathbf{4 a}-\mathbf{z}$.

Compound No.

Twenty six new oxime ethers $4 \mathbf{a}-\mathbf{z}$ were subjected to the docking study into the identified $\beta_{1}$ and $\beta_{2}$-adrenergic receptor active sites [17-19]. The docking technique using the Molegro Virtual Docker [20] is used to estimate the binding affinity between external ligands and structurally-known biological macromolecules, and hence, to predict the biological importance of these ligands [19,21]. The forces of interaction were declared and scoring functions were determined. Propranolol (a non-selective $\beta$-antagonist), cyanopindolol (selective $\beta_{1}$-antagonist) (Figure 2) and IPS 339 (selective $\beta 2$-antagonist) were used in this docking study as reference molecules. Snapshots for all prepared compounds were taken to reveal their molecular interactions (hydrogen, hydrophobic and/or ionic bonds) with the amino 
acids of $\beta_{1}$ and $\beta_{2}$ - receptor active sites. MolDock scores and the energy H-bond interactions between these compounds and receptor were calculated (Table 3). The results showed that all tested compounds have affinity for $\beta_{1}$ and $\beta_{2}$-adrenoceptors comparable to that of the reference compounds.

Figure 2. Chemical structures of propranolol and cyanopindolol.<smiles>CC(C)NCC(O)COc1cccc2ccccc12</smiles><smiles>CC(C)(C)NCC(O)COc1cccc2[nH]c(C#N)cc12</smiles>

Table 3. MolDock scores and hydrogen bond energy for compounds $4 \mathbf{a}-\mathbf{z}$ and reference compounds with the $\beta_{1}$ and $\beta_{2}$ receptors.

\begin{tabular}{|c|c|c|c|c|}
\hline \multirow{2}{*}{ Comp. } & \multicolumn{2}{|c|}{$\beta_{1}$-Receptor } & \multicolumn{2}{|c|}{$\beta_{2}$-Receptor } \\
\hline & MolDock Score & H-Bond E. & MolDock Score & H-Bond E. \\
\hline $4 a$ & -115.13 & -2.5 & -100.806 & -4.35757 \\
\hline $4 b$ & -122.697 & -4.58252 & -99.6442 & -2.42254 \\
\hline $4 c$ & -142.285 & -5.19707 & -105.6596 & -3.42955 \\
\hline 4d & -121.41 & -3.59754 & -86.4381 & -1.16172 \\
\hline $4 e$ & -127.903 & -3.96668 & -88.3577 & -0.04374 \\
\hline $4 f$ & -153.964 & -5 & -95.1594 & 0 \\
\hline $4 g$ & -125.653 & -2.5 & -92.7053 & -3.4013 \\
\hline $4 h$ & -124.044 & -2.83065 & -85.3741 & -0.69094 \\
\hline $4 i$ & -145.546 & -5.2648 & -90.616 & -2.98878 \\
\hline $4 j$ & -114.594 & -2.62113 & -79.1286 & 0 \\
\hline $4 k$ & -120.406 & -2.5 & -90.5964 & -2.63215 \\
\hline 41 & -155.836 & -6.1153 & -103.123 & -0.16123 \\
\hline $4 m$ & -125.091 & -3.98536 & -74.7409 & -0.97756 \\
\hline $4 n$ & -120.574 & -2.18542 & -73.6041 & -3.78389 \\
\hline 40 & -167.406 & -4.49787 & -91.127 & -2.5557 \\
\hline $4 p$ & -105.452 & -1.63375 & -113.5079 & -4.03237 \\
\hline $4 q$ & -114.132 & -2.62848 & -117.075 & -4.34578 \\
\hline $4 r$ & -143.244 & -2.15101 & -102.028 & -0.41247 \\
\hline $4 s$ & -108.994 & -1.50057 & -85.4672 & -3.26981 \\
\hline $4 t$ & -108.574 & -2.79887 & -84.921 & -0.78187 \\
\hline $4 u$ & -148.747 & -4.41468 & -100.887 & -0.03188 \\
\hline $4 v$ & -105.443 & -1.00129 & -84.9409 & -1.98422 \\
\hline $4 w$ & -105.034 & 0 & -85.1195 & -0.99963 \\
\hline $4 x$ & -139.094 & -4.07406 & -99.449 & -0.47872 \\
\hline $4 y$ & -152.107 & -5.54788 & -93.6354 & -2.4702 \\
\hline $4 z$ & -125.4 & -2.28939 & -84.4559 & -2.16834 \\
\hline Propranolol & -110.517 & -2.53142 & -88.1631 & -3.92249 \\
\hline IPS 339 & -104.212 & -2.91744 & -95.141 & -2.87182 \\
\hline Cyanopindolol & -129.204 & -10.7754 & -85.6768 & -2.98876 \\
\hline
\end{tabular}




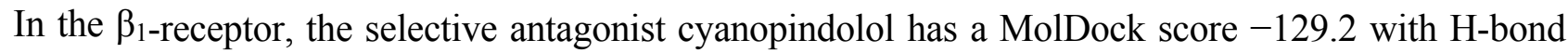
energy -10.7 and compounds $\mathbf{4 c}, \mathbf{4 f}, \mathbf{4 i}, \mathbf{4 l}, \mathbf{4 o}, \mathbf{4 r}, \mathbf{4} \mathbf{u}, \mathbf{4 x}$ and $\mathbf{4 y}$ have better MolDock scores than cyanopindolol. The best compound in this study was 4o, which has MolDock score of -167.4 and forms five hydrogen bonds with the amino acids Ser 211 and Tyr 207 in the active site of the receptor Moreover, hydrophobic interactions probably existed between compound 40 and Phe 201, Phe 216 and Phe 307 (Figure 3a). It is worth mentioning here that all tested compounds which have better MolDock score than the reference drug have in its structure the homoveratryl moiety which may increase the quality of binding between the ligands and receptor.

Figure 3. (a) Docking snapshot of compound 40 with $\beta_{1}$ receptor. (b) Docking snapshot of compound $\mathbf{4 q}$ with $\beta_{2}$ receptor. Compounds are represented as stick (thick lines) while the amino acids of the active site of the $\beta 2$-adrenoreceptor appear as (light) lines. Hydrogen atoms in both ligands and receptors have been omitted for clarity. Blue dashed lines indicate the hydrogen bonds.

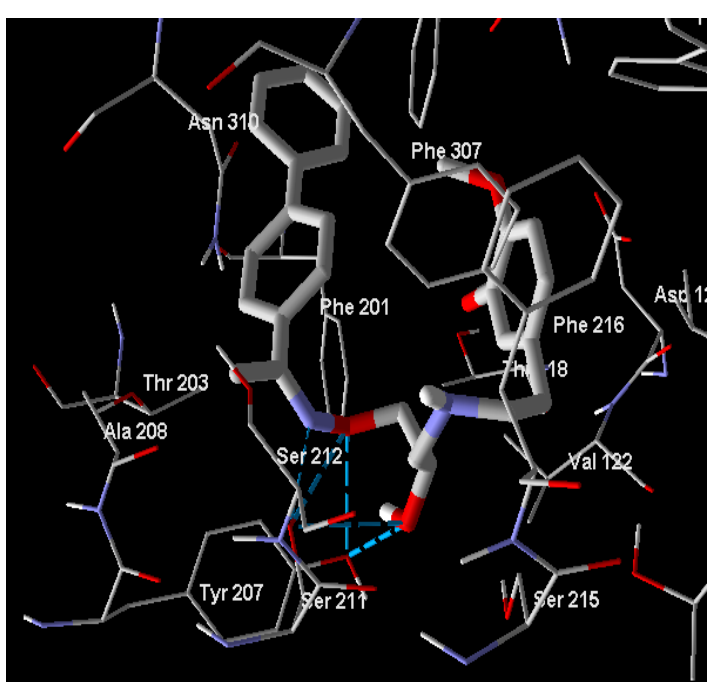

(a)

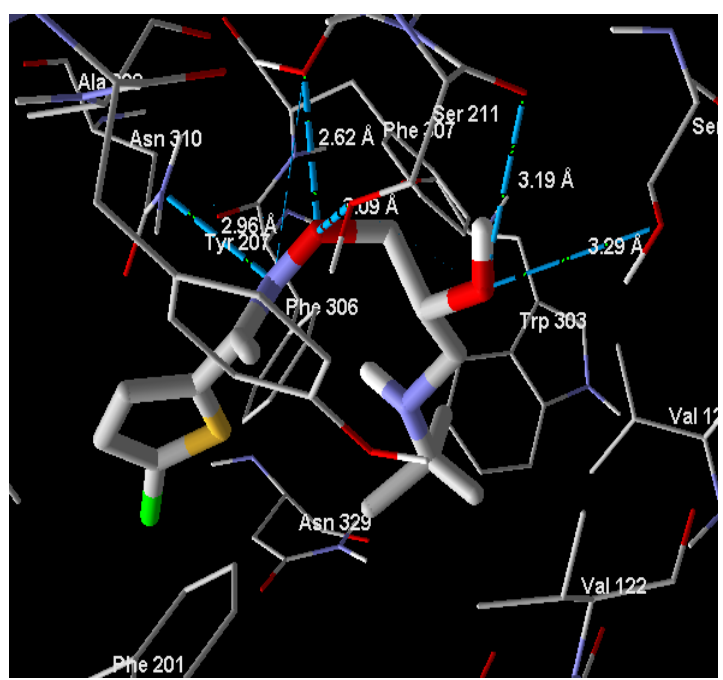

(b)

In the $\beta 2$-receptor, only six compounds $\mathbf{4 a}, \mathbf{4 b}, \mathbf{4 c}, \mathbf{4 l}, \mathbf{4} \mathbf{u}$ and $\mathbf{4 x}$ have slightly lower MolDock scores than the $\beta_{2}$-antagonist IPS 339 , while compounds $\mathbf{4 p}$ and $\mathbf{4 q}$ displayed the highest negative MolDock scores that indicated highest complementarity with $\beta_{2}$-adrenoceptors (Table 3 ).

Figure $3 b$ represents the snapshot for compound $\mathbf{4 q}$ and illustrates the different amino acids of the binding site that are involved in the compound-receptor interaction, where more hydrogen bonds and hydrophobic interactions were involved for this compound than others. Hydrogen bonding interactions were $\beta$-hydroxyl group in $\mathbf{4 q}$ with the peptide carbonyl group of Ser 215 (3.29 $\AA$ ) and with the free hydroxyl group of Ser 311 (3.19 Á), oxygen of the oxime moiety makes a hydrogen bond with Ser 211 and Ser 212 (3.09 and $2.62 \AA$, respectively) and the nitrogen of the oxime moiety also interacts with Asn 310 by hydrogen bonding (2.96 ̊́). Hydrophobic interactions may be found between the thiophene ring and the amino acids Phe 201 and Phe 306. 


\subsection{Biological Evaluation}

Blocking of the $\beta_{1}$-adrenoceptor prevents the increase in the heart rate and contractility caused by epinephrine, while $\beta_{2}$-adrenoceptor antagonism results in bronchospasms, uterine contraction and blood vessel spasms [22]. In the present investigation, certain newly synthesized compounds were subjected to in vitro biological testing for their blocking activity against $\beta_{1}$ and $\beta_{2}$-adrenoceptors, using isolated guinea pig atria and trachea, respectively, according to reported procedures [23-25]. Meanwhile, the agonistic activities at both receptors were also recorded.

\subsubsection{In Vitro Screening for $\beta_{1}$-Adrenoceptor Activity in Isolated Guinea Pig Atria}

Seventeen of the newly synthesized compounds were subjected to an in vitro biological screening for $\beta_{1}$-adrenoceptor activities using isolated atria of guinea pigs. The available results indicate that six of these compounds $(\mathbf{4 f}, \mathbf{4 i}, \mathbf{4}, \mathbf{4 r}, \mathbf{4} \mathbf{y}$ and $\mathbf{4 z}$ ), with 3,4-dimethoxyphenethyl groups at the terminal amine produced $22 \%-31 \%$ inhibition in the heart rate and $30 \%-55 \%$ inhibition in the contractility of the atria. Propranolol, the reference drug, produced 70 and $60 \%$ inhibition of the heart rate and contractility, respectively (Table 4).

Table 4. The percent inhibition in the heart rate and the contractility of the isolated guinea pig atria caused by the tested compounds.

\begin{tabular}{|c|c|c|c|c|c|}
\hline \multirow{2}{*}{$\begin{array}{c}\text { Compound } \\
\text { No. }\end{array}$} & \multicolumn{2}{|r|}{ B1-antagonist } & \multirow[b]{2}{*}{ Compd. No. } & \multicolumn{2}{|c|}{ B1-antagonist } \\
\hline & $\begin{array}{l}\text { \% inhibition } \\
\text { in heart rate }\end{array}$ & $\begin{array}{c}\% \text { inhibition in } \\
\text { contractility }\end{array}$ & & $\begin{array}{l}\text { \% inhibition } \\
\text { in heart rate }\end{array}$ & $\begin{array}{l}\% \text { Inhibition } \\
\text { in contractility }\end{array}$ \\
\hline $4 a$ & $3 \%$ & $0 \%$ & 40 & $1 \%$ & $0 \%$ \\
\hline $4 b$ & $2 \%$ & $0 \%$ & $4 p$ & $6 \%$ & $0 \%$ \\
\hline $4 c$ & $6 \%$ & $1 \%$ & $4 q$ & $2 \%$ & $0 \%$ \\
\hline $4 e$ & $0 \%$ & $0 \%$ & $4 r$ & $31 \%$ & $55 \%$ \\
\hline $4 f$ & $29 \%$ & $30 \%$ & $4 u$ & $2 \%$ & $0 \%$ \\
\hline $4 \mathrm{~g}$ & $2 \%$ & $0 \%$ & $4 x$ & $0 \%$ & $0 \%$ \\
\hline $4 i$ & $25 \%$ & $40 \%$ & $4 y$ & $26 \%$ & $35 \%$ \\
\hline $4 k$ & $3 \%$ & $1 \%$ & $4 z$ & $22 \%$ & $30 \%$ \\
\hline 41 & $28 \%$ & $45 \%$ & Propranolol & $70 \%$ & $60 \%$ \\
\hline
\end{tabular}

Replacement of the 3,4-dimethoxyphenethyl group with isopropyl or tert-butyl groups showed loss of $\beta_{1}$-adrenergic antagonist activity, as in $\mathbf{4 g}, \mathbf{4 e}, \mathbf{4 k}$ and $\mathbf{4 p}$. It is worth mentioning that the dimethoxy substitution on the phenethylamine was reported to be the preferable aralkylamine moiety required to achieve $\beta$-adrenergic receptor blocking activity [26]. On the other hand, compounds $\mathbf{4 c}, \mathbf{4 o}, \mathbf{4 u}$ and $\mathbf{4 x}$ contain 3,4-dimethoxyphenethylamine groups, but they did not show any $\beta_{1}$-adrenergic antagonistic activity. This might be explained by the structural difference on the other side of the oxime moiety, where the hydrophobic part might be improperly oriented to fit in the active site in the $\beta_{1}$-adrenergic receptor. Meanwhile, the study also indicated that none of the tested compounds has $\beta_{1}$-agonistic activity; whereby no increase in the heart rate or contractility of isolated guinea pig atria was noticed. 


\subsubsection{In Vitro Screening for $\beta 2$-Adrenoceptor Activity in Isolated Guinea pig Trachea}

Eleven of the newly synthesized compounds were subjected to an in vitro biological screening for $\beta_{2}$-adrenoceptor activities using isolated trachea of guinea pigs. The test depends principally on the relaxation effect of salbutamol on the pre-contracted tracheal muscle induced by acetylcholine. Compounds of potential antagonistic activity are expected to decrease the relaxation caused by salbutamol. The available results indicated that only two compounds, $\mathbf{4 p}$ and $\mathbf{4 q}$, decreased the salbutamol relaxation of pre-contracted tracheal strips, while the rest of the tested compounds did not show any $\beta_{2}$-antagonist activity. The results also showed the absence of agonistic activity for all tested compounds (Table 5).

Table 5. $\beta_{2}$-Adrenergic activities of the tested compounds.

\begin{tabular}{cccccc}
\hline Compound No. & $\boldsymbol{\beta 2}$-agonist & $\boldsymbol{\beta 2}$-antagonist & Compound No. & $\boldsymbol{\beta 2}$-agonist & $\boldsymbol{\beta 2}$-antagonist \\
\hline $\mathbf{4 a}$ & - & - & $\mathbf{4 p}$ & - & + \\
$\mathbf{4} \mathbf{e}$ & - & - & $\mathbf{4 q}$ & - & + \\
$\mathbf{4 g}$ & - & - & $\mathbf{4 r}$ & - & - \\
$\mathbf{4} \mathbf{i}$ & - & - & $\mathbf{4 t}$ & - & - \\
$\mathbf{4} \mathbf{k}$ & - & - & $\mathbf{4 v}$ & - & - \\
$\mathbf{4} \mathbf{m}$ & - & - & Propranolol & - & + \\
\hline
\end{tabular}

$(+)$ decreased relaxation, $(-)$ no effect.

Taken together, docking of twenty six compounds into the $\beta_{1}$ and $\beta_{2}$ adrenergic receptors has been studied, and the energy of interaction has been calculated. The energy of interaction revealed that compounds $\mathbf{4 p}$ and $\mathbf{4 q}$ indicated the highest complementarity with $\beta_{2}$-adrenoceptors (Table 3 ). These two compounds, in particular, decreased the salbutamol relaxation effect on guinea pig trachea, suggesting their $\beta_{2}$-antagonist activity. The structural characteristics that might differentiate compounds $\mathbf{4 p}$ and $\mathbf{4 q}$ from others is the presence of a chlorothiophene moiety in the hydrophobic region. Compounds with chlorothienyloxypropanolamine moieties were previously studied by El-Ashmawy et al. [27] for their $\beta$-blocking and partial agonist activity, but no tests were done with $\beta_{2}$-receptors. Compound $4 \mathbf{r}$ also has the same hydrophobic chlorothienyl moiety, however, the presence of a bulky homoveratrylamine portion might explain its inactivity at the $\beta_{2}$-adrenoceptors. It is worth mentioning that this compound (4r) displayed the highest percentage inhibition on $\beta 1$-adrenoceptor $(55 \%$ inhibition of the contractility) (Table 4$)$. These findings confirm the significance of a homoveratrylamine portion for $\beta_{1}$ receptors, especially in view of the data from the $\beta_{1}$-adrenergic receptor docking study (Table 4); and indicate the importance of the presence of a chlorothiophene moiety in the hydrophobic region for best complementarity with $\beta_{2}$ receptors.

\section{Experimental}

\subsection{General Information}

Melting points $\left({ }^{\circ} \mathrm{C}\right)$ were recorded using a Fisher-Johns melting point apparatus and are uncorrected. ${ }^{1}$ H-NMR spectra were obtained on FT-NMR spectrometer $(200 \mathrm{MHz})$ Gemini Varian using TMS as internal standard (chemical shifts in ppm, $\delta$ units). MS analyses were performed on JEOL JMS-600H 
spectrometer. All the ketones used were commercially available from Sigma-Aldrich Chemical Company (Munich, Germany), except benzophenone and acetophenone that were purchased from ElNasr Pharmaceutical Chemicals Company, Cairo, Egypt. Oximes 2a-j were prepared following the procedures reported in the literature [3,7-14]. Two of the oxirane compounds $\mathbf{3 i}$ and $\mathbf{3 j}$ were previously reported in the literature [14].

\subsubsection{General Procedure for Synthesis of $O$-Oxiran-2-ylmethyl Oximes 3a-h}

A mixture of the appropriate oxime $\mathbf{2 a}-\mathbf{h}(0.01 \mathrm{~mol})$ and $\mathrm{NaH}(0.26 \mathrm{~g}, 0.011 \mathrm{~mol})$ was stirred in dry DMF $(30 \mathrm{~mL})$ at room temperature for $10 \mathrm{~min}$. A solution of epichlorohydrin $(1.1 \mathrm{~g}, 0.01 \mathrm{~mol})$ in dry DMF $(5 \mathrm{~mL})$ was added dropwise over a period of $10 \mathrm{~min}$. The resulting mixture was further stirred for $36 \mathrm{~h}$ at room temperature (TLC). The reaction mixture was poured in ice water $(200 \mathrm{~mL})$ and extracted with chloroform $(3 \times 100 \mathrm{~mL})$. The organic layers were collected, washed twice with brine solution, dried over anhydrous $\mathrm{MgSO}_{4}$, and the chloroform was evaporated under reduced pressure to obtain the desired compounds in pure form.

Dicyclohexylmethanone O-oxiran-2-yl methyl oxime (3a). Yield 55\%; M.p. $120-122{ }^{\circ} \mathrm{C}$; ${ }^{1} \mathrm{H}-\mathrm{NMR}$ $\left(\mathrm{CDCl}_{3}\right) \delta 1.15-1.70\left(\mathrm{~m}, 20 \mathrm{H}, 10 \mathrm{CH}_{2}\right.$ in cyclohexane), 2.21-2.44 (m, 2H, 2CHC=NO), 2.54-2.90 (m, 3H, $\mathrm{CH}_{2}$ and $\mathrm{CH}$ oxirane), $3.85\left(\mathrm{dd}, 1 \mathrm{H}, J=11.2,6.4 \mathrm{~Hz}, \mathrm{OCH}_{2}\right), 4.17\left(\mathrm{dd}, 1 \mathrm{H}, J=11.4,2.6 \mathrm{~Hz}, \mathrm{OCH}_{2}\right)$. MS $m / z(\%) ; 266$ (10.6, $\left.\mathrm{M}^{+}+1\right), 176$ (63.2), 147 (100), 95 (48.65).

Cyclohexyl(phenyl)methanone O-oxiran-2-yl methyl oxime (3b). Yield 60\%; oil; ${ }^{1} \mathrm{H}-\mathrm{NMR}\left(\mathrm{CDCl}_{3}\right) \delta$ 1.25-1.70 (m, 10H, 5CH $\mathrm{CH}_{2}$ in cyclohexane), 2.15-2.41 (m, 1H, CHC=NO), 2.74-3.01 (m, 3H, $\mathrm{CH}_{2}, \mathrm{CH}$ oxirane), $4.11\left(\mathrm{dd}, 1 \mathrm{H}, J=11.5,6.4 \mathrm{~Hz}, \mathrm{OCH}_{2}\right), 4.40\left(\mathrm{dd}, 1 \mathrm{H}, J=11.6,3.0 \mathrm{~Hz}, \mathrm{OCH}_{2}\right), 7.55(\mathrm{t}, 3 \mathrm{H}$, Ar-H), 7.91 (d, 2H, J = 7.0, 2,6 Ar-H). MS m/z (\%); 260 (4.55, $\left.\mathrm{M}^{+}+1\right), 186$ (100), 104 (78), 77 (61.62).

Phenyl(Pyridin-2-yl)methanone O-oxiran-2-yl methyl oxime (3c). Yield 62\%; oil; ${ }^{1} \mathrm{H}-\mathrm{NMR}\left(\mathrm{CDCl}_{3}\right) \delta$ 2.64-2.90 (m, 3H, $\mathrm{CH}_{2}, \mathrm{CH}$ oxirane), $3.91\left(\mathrm{dd}, 1 \mathrm{H}, J=11.4,6.1 \mathrm{~Hz}, \mathrm{OCH}_{2}\right), 4.31(\mathrm{dd}, 1 \mathrm{H}, J=11.8$, $\left.2.9 \mathrm{~Hz}, \mathrm{OCH}_{2}\right), 7.44-7.68(\mathrm{~m}, 5 \mathrm{H}, \mathrm{Ar}-\mathrm{H}$ and pyridinyl-H), 7.79 (d, 2H, J = 7.0, 2,6-Ar-H), 7.95 (d, 1H, $J=7.5$, pyridinyl-H) $8.51(\mathrm{~d}, 1 \mathrm{H}, J=8.0$, pyridinyl-H).

1-(Naphthalen-2-yl)ethanone O-oxiran-2-yl methyl oxime (3d). Yield 70\%; M.p. 40-42 ${ }^{\circ} \mathrm{C} ;{ }^{1} \mathrm{H}-\mathrm{NMR}$ $\left(\mathrm{CDCl}_{3}\right) \delta$ 2.54-2.77 (m, 3H, $\mathrm{CH}_{2}, \mathrm{CH}$ oxirane), $2.95\left(\mathrm{~s}, 3 \mathrm{H}, \mathrm{CH}_{3}\right), 3.86(\mathrm{dd}, 1 \mathrm{H}, J=11.5,6.5 \mathrm{~Hz}$, $\left.\mathrm{OCH}_{2}\right), 4.17$ (dd, $\left.1 \mathrm{H}, J=11.6,2.6 \mathrm{~Hz}, \mathrm{OCH}_{2}\right), 7.42-7.71$ (m, 7H, Ar-H). MS m/z (\%); $242\left(8.06, \mathrm{M}^{+}+1\right)$, 154 (63.2), 127 (100), 87 (48.65).

1-(Biphenyl-4-yl)ethanone O-oxiran-2-yl methyl oxime (3e). Yield 70\%; M.p. $72-74{ }^{\circ} \mathrm{C}$; ${ }^{1} \mathrm{H}-\mathrm{NMR}$ $\left(\mathrm{CDCl}_{3}\right) \delta 2.18\left(\mathrm{~s}, 3 \mathrm{H}, \mathrm{CH}_{3}\right), 2.47-2.91\left(\mathrm{~m}, 3 \mathrm{H}, \mathrm{CH}_{2}, \mathrm{CH}\right.$ oxirane), $3.74(\mathrm{dd}, 1 \mathrm{H}, J=11.2,6.6 \mathrm{~Hz}$, $\left.\mathrm{OCH}_{2}\right), 3.96\left(\mathrm{dd}, 1 \mathrm{H}, J=11.4,2.9 \mathrm{~Hz}, \mathrm{OCH}_{2}\right), 7.45\left(\mathrm{t}, 3 \mathrm{H}, J=8.5,3^{\prime}, 4^{\prime}, 5^{\prime}-\mathrm{Ar}-\mathrm{H}\right), 7.65(\mathrm{~d}, 2 \mathrm{H}$, $\left.J=7.5,2^{\prime}, 6^{\prime}-\mathrm{Ar}-\mathrm{H}\right), 7.98$ (d, 2H, $\left.J=8.5 \mathrm{Ar}-\mathrm{H}\right), 8.24$ (d, 2H, $\left.J=8.5 \mathrm{Ar}-\mathrm{H}\right)$.

1-(5-Chlorothiophen-2-yl)ethanone O-oxiran-2-yl methyl oxime (3f). Yield 65\%; oil; ${ }^{1} \mathrm{H}-\mathrm{NMR}$ $\left(\mathrm{CDCl}_{3}\right) \delta 2.16\left(\mathrm{~s}, 3 \mathrm{H}, \mathrm{CH}_{3}\right), 2.56-3.04\left(\mathrm{~m}, 3 \mathrm{H}, \mathrm{CH}_{2}, \mathrm{CH}\right.$ oxirane), $4.16(\mathrm{dd}, 1 \mathrm{H}, J=11.3,6.5 \mathrm{~Hz}$, $\left.\mathrm{OCH}_{2}\right), 4.30\left(\mathrm{dd}, 1 \mathrm{H}, J=11.2,2.7 \mathrm{~Hz}, \mathrm{OCH}_{2}\right), 7.15$ (d, $1 \mathrm{H}, J=8.0,3$-thiophene-H), $7.41(\mathrm{~d}, 1 \mathrm{H}$, $J=8.5$, 4-thiophene-H). 
1,3,3-Trimethylbicyclo[2.2.1] heptan-2-one O-oxiran-2-yl methyl oxime (3g). Yield 45\%; oil; ${ }^{1} \mathrm{H}-\mathrm{NMR}$ $\left(\mathrm{CDCl}_{3}\right) \delta 1.30\left(\mathrm{~s}, 6 \mathrm{H}, 2 \mathrm{CH}_{3}\right), 1.35\left(\mathrm{~s}, 3 \mathrm{H}, \mathrm{CH}_{3}\right), 1.52-1.70(\mathrm{~m}, 7 \mathrm{H}$, bicyclic moiety-H), 2.41-2.70 (m, 3H, $\mathrm{CH}_{2}, \mathrm{CH}$ oxirane), $3.75\left(\mathrm{dd}, 1 \mathrm{H}, J=11.0,6.1 \mathrm{~Hz}, \mathrm{OCH}_{2}\right), 3.94(\mathrm{dd}, 1 \mathrm{H}, J=11.5,2.4 \mathrm{~Hz}$, $\left.\mathrm{OCH}_{2}\right)$. MS m/z (\%); 224 (5.16, $\left.\mathrm{M}^{+}+1\right), 150$ (56.62), 136 (100), 77 (35.04).

5-Methoxy-3,4-dihydronaphthalen-1(2H)-one O-oxiran-2-yl methyl oxime (3h). Yield 67\%; oil; ${ }^{1} \mathrm{H}-\mathrm{NMR}\left(\mathrm{CDCl}_{3}\right) \delta 2.17-2.91\left(\mathrm{~m}, 9 \mathrm{H}, 3 \mathrm{CH}_{2}\right.$ of dihydronaphthalene, $\mathrm{CH}_{2}$ oxirane, $\mathrm{CH}$ oxirane), $3.78\left(\mathrm{~s}, 3 \mathrm{H}, \mathrm{OCH}_{3}\right), 3.85\left(\mathrm{dd}, 1 \mathrm{H}, J=11.2,6.0 \mathrm{~Hz}, \mathrm{OCH}_{2}\right), 4.1\left(\mathrm{dd}, 1 \mathrm{H}, J=11.5,2.7 \mathrm{~Hz}, \mathrm{OCH}_{2}\right), 7.14$ (d, $1 \mathrm{H}, J=8.0,6-\mathrm{Ar}-\mathrm{H}), 7.35$ (t, $1 \mathrm{H}, J=8.5,7-\mathrm{Ar}-\mathrm{H}), 7.56$ (d, 1H, $J=8.5,8-\mathrm{Ar}-\mathrm{H})$.

\subsubsection{General Procedure for Synthesis of $O$-(3-Alkylamino-2-hydroxypropyl)oxime Derivatives $\mathbf{4 a}-\mathbf{z}$}

A mixture of the appropriate oxirane $3 \mathbf{a}-\mathbf{j}(0.01 \mathrm{~mol})$ and the appropriate amine (isopropylamine, tert-butylamine or homoveratrylamine, $0.04 \mathrm{~mol})$ in anhydrous toluene $(10 \mathrm{~mL})$ was heated in sealed tube at $120{ }^{\circ} \mathrm{C}$ for $24 \mathrm{~h}$ (TLC). After cooling, the solution was evaporated under reduced pressure to give an oily residue. The obtained product was dissolved in diethylether, and treated with oxalic acid $(0.45 \mathrm{~g}, 0.005 \mathrm{~mol})$ in acetone or ethereal $\mathrm{HCl}$ to give either the oxalate or hydrochloride salt. The separated solid was collected by filtration, washed with diethyl ether, dried and crystallized from ethanol.

Dicyclohexylmethanone O-2-hydroxy-3-(isopropylamino)propyl oxime, hydrogen oxalate (4a). Yield 63\%; M.p. $123-125{ }^{\circ} \mathrm{C}$; ${ }^{1} \mathrm{H}-\mathrm{NMR}$ (free base in $\mathrm{CDCl}_{3}$ ) $\delta 1.06\left(\mathrm{~d}, 6 \mathrm{H}, J=6.5,2 \mathrm{CH}_{3}\right.$ ), $1.52-1.77$ (m, 20H, 10CH $\mathrm{CH}_{2}$ cyclohexane), 2.42-2.65 (m, 2H, 2Cㅡㄷ $\left.=\mathrm{NO}\right), 2.95-3.12\left(\mathrm{~m}, 3 \mathrm{H}, \underline{\mathrm{C}}_{2} \mathrm{~N}, \mathrm{C} \underline{\mathrm{H}}\left(\mathrm{CH}_{3}\right)_{2}\right)$, 3.4-3.7 (m, 3H, $\mathrm{CHOH}, \mathrm{OH}$ and $\mathrm{NH}$ exchangeable with $\left.\mathrm{D}_{2} \mathrm{O}\right), 3.95(\mathrm{dd}, 1 \mathrm{H}, J=10.8,6.4 \mathrm{~Hz}$, $\left.\mathrm{OCH}_{2}\right), 4.15\left(\mathrm{dd}, 1 \mathrm{H}, J=11.2,2.8 \mathrm{~Hz}, \mathrm{OCH}_{2}\right)$. MS m/z (\%); $326\left(6.19, \mathrm{M}^{+}+2\right), 325\left(6.82, \mathrm{M}^{+}+1\right)$, 210 (41.72), 98 (100), 83 (34.55), 72 (81.95).

Dicyclohexylmethanone O-3-(tert-butylamino)-2-hydroxypropyl oxime, hydrogen oxalate (4b). Yield 55\%; M.p. $117-119{ }^{\circ} \mathrm{C}$; ${ }^{1} \mathrm{H}-\mathrm{NMR}$ (free base in $\left.\mathrm{CDCl}_{3}\right) \delta 1.46\left(\mathrm{~s}, 9 \mathrm{H}, 3 \mathrm{CH}_{3}\right), 1.59-1.72(\mathrm{~m}, 20 \mathrm{H}$, $10 \mathrm{CH}_{2}$ cyclohexane), 2.41-2.55 (m, $\left.2 \mathrm{H}, \mathrm{CHC}=\mathrm{NO}\right), 2.81-2.98\left(\mathrm{~m}, 2 \mathrm{H}, \underline{\mathrm{CH}_{2}} \underline{\mathrm{NH}}\right), 3.35-3.54(\mathrm{~m}, 3 \mathrm{H}$, $\mathrm{C} \underline{\mathrm{HOH}}, \mathrm{OH}$ and $\mathrm{NH}$ exchangeable with $\left.\mathrm{D}_{2} \mathrm{O}\right), 4.12\left(\mathrm{dd}, 1 \mathrm{H}, J=11.2,6.2 \mathrm{~Hz}, \mathrm{OCH}_{2}\right), 4.26(\mathrm{dd}, 1 \mathrm{H}$, $\left.J=11.8,2.1 \mathrm{~Hz}, \mathrm{OCH}_{2}\right)$. MS $m / z(\%) ; 340\left(9.9, \mathrm{M}^{+}+2\right), 339$ (5.04, $\left.\mathrm{M}^{+}+1\right), 225$ (38.09), 113 (100), $86(10.24), 72(76.36)$.

Dicyclohexylmethanone O-3-(3,4-dimethoxyphenethylamino)-2-hydroxypropyl oxime, hydrogen oxalate (4c). Yield 50\%; M.p. 174-176 ${ }^{\circ} \mathrm{C}$; $1 \mathrm{H}-\mathrm{NMR}$ (free base in $\left.\mathrm{CDCl}_{3}\right) \delta 1.5-1.8\left(\mathrm{~m}, 20 \mathrm{H}, 10 \mathrm{CH}_{2}\right.$ cyclohexane), 2.4-2.9 (m, 8H, 2CHC=NO, $\left.\underline{\mathrm{C}}_{2} \mathrm{NHC}_{2} \underline{\mathrm{C}}_{2}\right), 3.50-3.71(\mathrm{~m}, 3 \mathrm{H}, \mathrm{C} \underline{\mathrm{HOH}}, \mathrm{OH}$ and NH exchangeable with $\left.\mathrm{D}_{2} \mathrm{O}\right), 3.97\left(\mathrm{~s}, 6 \mathrm{H}, 2 \mathrm{OCH}_{3}\right), 4.08\left(\mathrm{dd}, 1 \mathrm{H}, J=10.8,6.0 \mathrm{~Hz}, \mathrm{OCH}_{2}\right), 4.24(\mathrm{dd}, 1 \mathrm{H}$, $\left.J=11.5,2.5 \mathrm{~Hz}, \mathrm{OCH}_{2}\right), 6.82(\mathrm{~d}, 1 \mathrm{H}, J=9.0, \mathrm{Ar}-\mathrm{H}), 7.01$ (s, 1H, Ar-H), 7.21 (d, 1H, $\left.J=8.5, \mathrm{Ar}-\mathrm{H}\right)$.

Cyclohexyl(phenyl)methanone O-2-hydroxy-3-(isopropylamino)propyl oxime, hydrochloride (4d). Yield 70\%; M.p. $157-159{ }^{\circ} \mathrm{C}$; ${ }^{1} \mathrm{H}-\mathrm{NMR}$ (free base in $\left.\mathrm{CDCl}_{3}\right) \delta 1.15\left(\mathrm{~d}, 6 \mathrm{H}, J=6.5,2 \mathrm{CH}_{3}\right.$ ), $1.52-1.77$ (m, $10 \mathrm{H}, 5 \mathrm{CH}_{2}$ cyclohexane), 2.32-2.41 (m, 1H, $\left.\underline{\mathrm{HC}}=\mathrm{NO}\right), 2.77-2.89\left(\mathrm{~m}, 3 \mathrm{H}, \mathrm{C}_{2} \underline{\mathrm{N}}, \mathrm{C} \underline{\mathrm{H}}\left(\mathrm{CH}_{3}\right)_{2}\right)$, 3.45-3.62 (m, 3H, $\underline{\mathrm{HOH}}, \mathrm{OH}$ and $\mathrm{NH}$ exchangeable with $\left.\mathrm{D}_{2} \mathrm{O}\right), 3.98\left(\mathrm{dd}, 1 \mathrm{H}, J=11.2,6.1 \mathrm{~Hz}, \mathrm{OCH}_{2}\right)$, 
4.12 (dd, 1H, $\left.J=11.6,2.7 \mathrm{~Hz}, \mathrm{OCH}_{2}\right), 7.45$ (m, 5H, Ar-H). MS m/z (\%); $320\left(6.94, \mathrm{M}^{+}+2\right), 319$ (3.75, $\left.\mathrm{M}^{+}+1\right), 188$ (68.77), 104 (70.80), 72 (100).

Cyclohexyl(phenyl)methanone O-3-(tert-butylamino)-2-hydroxypropyl oxime, hydrochloride (4e). Yield 55\%; M.p. 107-109 ${ }^{\circ} \mathrm{C}$; ${ }^{1} \mathrm{H}-\mathrm{NMR}$ (free base in DMSO- $\left.d_{6}\right) \delta 1.21\left(\mathrm{~s}, 9 \mathrm{H}, 3 \mathrm{CH}_{3}\right.$ ), 1.47-1.68 (m, $10 \mathrm{H}, 5 \mathrm{CH}_{2}$ cyclohaxane), 2.45-2.62 (m, $\left.1 \mathrm{H}, \mathrm{CHC}=\mathrm{NO}\right), 3.1-3.4\left(\mathrm{~m}, 2 \mathrm{H}, \mathrm{CH}_{2} \mathrm{~N}\right), 3.44-3.68(\mathrm{~m}, 3 \mathrm{H}$, $\mathrm{C} \underline{\mathrm{HOH}}, \mathrm{OH}$ and $\mathrm{NH}$ exchangeable with $\left.\mathrm{D}_{2} \mathrm{O}\right), 3.88\left(\mathrm{dd}, 1 \mathrm{H}, J=11.0,6.5 \mathrm{~Hz}, \mathrm{OCH}_{2}\right), 4.19(\mathrm{dd}, 1 \mathrm{H}$, $\left.J=11.3,2.5 \mathrm{~Hz}, \mathrm{OCH}_{2}\right), 7.55$ (m, 5H, Ar-H). MS m/z (\%); $334\left(7.80, \mathrm{M}^{+}+2\right), 333\left(5.10, \mathrm{M}^{+}+1\right), 146$ (50.15), 172 (73.08), 72 (100).

Cyclohexyl(phenyl)methanone O-3-(3,4-dimethoxyphenethyl amino)-2-hydroxypropyl oxime, hydrogen oxalate (4f). Yield 48\%; M.p. $140-142{ }^{\circ} \mathrm{C} ;{ }^{1} \mathrm{H}-\mathrm{NMR}$ (free base in $\mathrm{CDCl}_{3}$ ) $\delta 1.40-1.70$ (m, $10 \mathrm{H}, 5 \mathrm{CH}_{2}$ cyclohaxane), 2.38-2.65 (m, 7H, CHC=NO, $\left.\underline{\mathrm{C}}_{2} \mathrm{NHC}_{2} \underline{\mathrm{CH}}_{2}\right), 3.40-3.65(\mathrm{~m}, 3 \mathrm{H}, \mathrm{C} \underline{\mathrm{HOH}}$, $\mathrm{OH}$ and $\mathrm{NH}$ exchangeable with $\left.\mathrm{D}_{2} \mathrm{O}\right), 3.8\left(\mathrm{~s}, 6 \mathrm{H}, 2 \mathrm{OCH}_{3}\right), 4.00\left(\mathrm{dd}, 1 \mathrm{H}, J=11.5,6.2 \mathrm{~Hz}, \mathrm{OCH}_{2}\right)$, $4.18\left(\mathrm{dd}, 1 \mathrm{H}, J=11.6,2.6 \mathrm{~Hz}, \mathrm{OCH}_{2}\right), 6.9$ (d, $1 \mathrm{H}, J=8.5$, Ar-H homoveratryl), $7.2(\mathrm{~d}, 1 \mathrm{H}, J=8.5$, Ar-H homoveratryl), 7.2 (s, 1H, Ar-H homoveratryl), 7.6 (m, 5H, Ar-H). MS m/z (\%); 442 (10.12, $\left.\mathrm{M}^{+}+2\right), 441$ (9.33, $\left.\mathrm{M}^{+}+1\right), 289$ (27.80) 186 (41.90), 104 (100).

Phenyl(pyridin-2-yl)methanone O-2-hydroxy-3-(isopropylamino)propyl oxime, hydrochloride (4g). Yield 69\%; M.p. 191-193 ${ }^{\circ} \mathrm{C}$; ${ }^{1} \mathrm{H}-\mathrm{NMR}$ (free base in DMSO- $\left.d_{6}\right) \delta 1.44\left(\mathrm{~d}, 6 \mathrm{H}, J=6.5,2 \mathrm{CH}_{3}\right.$ ), 2.95-3.12 (m, 3H, $\left.\underline{\mathrm{C}}_{2} \mathrm{~N}, \mathrm{C} \underline{\mathrm{H}}\left(\mathrm{CH}_{3}\right)_{2}\right), 3.6-3.8\left(\mathrm{~m}, 3 \mathrm{H}, \mathrm{CHOH}, \mathrm{OH}\right.$ and $\mathrm{NH}$ exchangeable with $\left.\mathrm{D}_{2} \mathrm{O}\right)$, $4.05\left(\mathrm{dd}, 1 \mathrm{H}, J=11.5,6.0 \mathrm{~Hz}, \mathrm{OCH}_{2}\right), 4.24\left(\mathrm{dd}, 1 \mathrm{H}, J=11.0,2.5 \mathrm{~Hz}, \mathrm{OCH}_{2}\right), 7.4-7.7(\mathrm{~m}, 5 \mathrm{H}$, 3,4,5-Ar-H and 3,5-pyridinyl-H), 7.9-8.1 (m, 3H, 2,6-Ar-H, 4-pyridinyl-H), 8.5 (d, 1H, $J=8.0$, 6-pyridinyl-H). MS m/z (\%); 315 (0.13, $\left.\mathrm{M}^{+}+2\right), 314\left(0.55, \mathrm{M}^{+}+1\right), 313\left(0.20, \mathrm{M}^{+}\right), 198(100), 181$ (40.71), 72 (44.96).

Phenyl(pyridin-2-yl)methanone O-3-(tert-butylamino)-2-hydroxypropyl oxime, hydrochloride (4h). Yield 55\%; M.p. $180-182{ }^{\circ} \mathrm{C}$; ${ }^{1} \mathrm{H}-\mathrm{NMR}$ (free base in DMSO- $\left.d_{6}\right) \delta 1.27\left(\mathrm{~s}, 9 \mathrm{H}, 3 \mathrm{CH}_{3}\right), 2.94-3.08(\mathrm{~m}$, $\left.3 \mathrm{H}, \mathrm{CH}_{2} \mathrm{~N}\right), 3.5-3.8\left(\mathrm{~m}, 3 \mathrm{H}, \mathrm{CHOH}, \mathrm{OH}\right.$ and $\mathrm{NH}$ exchangeable with $\left.\mathrm{D}_{2} \mathrm{O}\right), 4.14(\mathrm{dd}, 1 \mathrm{H}, J=11.5,6.2 \mathrm{~Hz}$, $\mathrm{OCH}_{2}$ ), 4.33 (dd, $1 \mathrm{H}, J=11.6,2.8 \mathrm{~Hz}, \mathrm{OCH}_{2}$ ), 7.6-7.8 (m, 5H, 3,4,5-Ar-H and 3,5- pyridinyl-H), 8.0-8.2 (m, 3H, 2,6-Ar-H, 4-pyridinyl-H), 8.5 (d, $1 \mathrm{H}, J=8,6$-pyridinyl-H). MS $m / z(\%) ; 329\left(5.38, \mathrm{M}^{+}+2\right)$, 328 (1.35, $\left.\mathrm{M}^{+}+1\right), 198$ (100), 183 (70.91), 86 (46.49), 78 (42.53).

Phenyl (pyridin-2-yl)methanone O-3-(3,4-dimethoxyphenethyl amino)-2-hydroxypropyl oxime, hydrogen oxalate (4i). Yield 50\%; M.p. $177-179{ }^{\circ} \mathrm{C}$; ${ }^{1} \mathrm{H}-\mathrm{NMR}$ (free base in $\mathrm{CDCl}_{3}$ ) $\delta 2.4-2.9$ (m, $\left.6 \mathrm{H}, \underline{\mathrm{C}}_{2} \mathrm{NHCH}_{2} \mathrm{C}_{2}\right), 3.50-3.85\left(\mathrm{~m}, 3 \mathrm{H}, \mathrm{CHOH}, \mathrm{OH}\right.$ and $\mathrm{NH}$ exchangeable with $\left.\mathrm{D}_{2} \mathrm{O}\right), 3.97(\mathrm{~s}, 6 \mathrm{H}$, $\left.2 \mathrm{OCH}_{3}\right), 4.10\left(\mathrm{dd}, 1 \mathrm{H}, J=11.5,6.0 \mathrm{~Hz}, \mathrm{OCH}_{2}\right), 4.22\left(\mathrm{dd}, 1 \mathrm{H}, J=11.0,2.8 \mathrm{~Hz}, \mathrm{OCH}_{2}\right), 6.8(\mathrm{~d}, 2 \mathrm{H}$, 5,6-Ar-H homoveratryl), $7.1(\mathrm{~s}, 1 \mathrm{H}, 2-\mathrm{Ar}-\mathrm{H}$ homoveratryl), 7.4-7.7 (m, 5H, 3,4,5-Ar-H and 3,5- pyridinyl-H), 7.9 (m, 3H, 2,6-Ar-H, 4-pyridinyl-H), 8.5 (d, 1H, J=8.5, 6-pyridinyl-H).

1-(Naphthalen-2-yl)ethanone O-2-hydroxy-3-(isopropylamino)propyl oxime, hydrochloride (4j). Yield 67\%; M.p. $117-119{ }^{\circ} \mathrm{C}$; ${ }^{1} \mathrm{H}-\mathrm{NMR}$ (free base in $\left.\mathrm{CDCl}_{3}\right) \delta 1.61\left(\mathrm{~d}, 6 \mathrm{H}, J=6.5,2 \mathrm{CH}_{3}\right), 2.20$ (s, $\left.3 \mathrm{H}, \mathrm{CH}_{3}\right), 2.7-2.9\left(\mathrm{~m}, 2 \mathrm{H}, \mathrm{C}_{2} \mathrm{NH}\right), 3.44-3.65(\mathrm{~m}, 3 \mathrm{H}, \mathrm{CHOH}, \mathrm{OH}$ and $\mathrm{NH}$ exchangeable with 
$\left.\mathrm{D}_{2} \mathrm{O}\right), 4.42\left(\mathrm{dd}, 1 \mathrm{H}, J=11.6,6.4 \mathrm{~Hz}, \mathrm{OCH}_{2}\right), 4.57\left(\mathrm{dd}, 1 \mathrm{H}, J=11.2,2.4 \mathrm{~Hz}, \mathrm{OCH}_{2}\right), 7.65(\mathrm{t}, 2 \mathrm{H}$, $J=8.0,6,7-\mathrm{Ar}-\mathrm{H}), 8.06(\mathrm{~d}, 4 \mathrm{H}, J=7.5,3,4,5,8-\mathrm{Ar}-\mathrm{H}), 8.45$ (s, $1 \mathrm{H}, 1-\mathrm{Ar}-\mathrm{H}) . \mathrm{MS} \mathrm{m} / z(\%) ; 300$ (1.60, $\left.\mathrm{M}^{+}\right), 168$ (6.54), 127 (21.09), 73 (91.92), (100), 60 (55.21).

1-(Naphthalen-2-yl)ethanone O-3-(tert-butylamino)-2-hydroxypropyl oxime, hydrochloride (4k). Yield 59\%; M.p. $120-122{ }^{\circ} \mathrm{C}$; ${ }^{1} \mathrm{H}-\mathrm{NMR}$ (free base in $\left.\mathrm{CDCl}_{3}\right) \delta 1.18\left(\mathrm{~s}, 9 \mathrm{H}, 3 \mathrm{CH}_{3}\right), 2.38\left(\mathrm{~s}, 3 \mathrm{H}, 3 \mathrm{CH}_{3}\right)$, 3.0-3.4 (m, 2H, $\left.\underline{\mathrm{C}}_{2} \mathrm{NH}\right), 3.4-3.75$ (m, 3H, $\mathrm{CHOH}, \mathrm{OH}$ and $\mathrm{NH}$ exchangeable with $\mathrm{D}_{2} \mathrm{O}$ ), 4.24 (dd, $\left.1 \mathrm{H}, J=11.3,6.2 \mathrm{~Hz}, \mathrm{OCH}_{2}\right), 4.38\left(\mathrm{dd}, 1 \mathrm{H}, J=11.8,2.2 \mathrm{~Hz}, \mathrm{OCH}_{2}\right), 7.56(\mathrm{t}, 2 \mathrm{H}, J=8.56,7-\mathrm{Ar}-\mathrm{H})$, $8(\mathrm{~d}, 4 \mathrm{H}, J=7.5,3,4,5,8-\mathrm{Ar}-\mathrm{H}), 8.4$ (s, 1H, 1-Ar-H). MS m/z (\%); $316\left(9.25, \mathrm{M}^{+}+2\right), 315(7.18$, $\left.\mathrm{M}^{+}+1\right), 167$ (84.11), 127 (60.13), 86 (100), 58 (43.86).

1-(Naphthalen-2-yl)ethanone O-3-(3,4-dimethoxyphenethyl amino)-2-hydroxypropyl oxime, hydrogen oxalate (4I). Yield 50\%; M.p. 181-183 ${ }^{\circ} \mathrm{C} ;{ }^{1} \mathrm{H}-\mathrm{NMR}$ (free base in $\left.\mathrm{CDCl}_{3}\right) \delta 2.10\left(\mathrm{~s}, 3, \mathrm{CH}_{3}\right), 3.1-3.4(\mathrm{~m}$, $\left.6 \mathrm{H}, \mathrm{C}_{2}{ }_{2} \mathrm{NHC}_{2} \underline{\mathrm{C}}_{2}\right), 3.3 .75\left(\mathrm{~m}, 3 \mathrm{H}, \mathrm{C} \underline{\mathrm{HOH}}, \mathrm{OH}\right.$ and $\mathrm{NH}$ exchangeable with $\left.\mathrm{D}_{2} \mathrm{O}\right), 3.8(\mathrm{~s}, 6 \mathrm{H}$, $\left.2 \mathrm{OCH}_{3}\right), 4.15\left(\mathrm{dd}, 1 \mathrm{H}, J=11.5,6.2 \mathrm{~Hz}, \mathrm{OCH}_{2}\right), 4.30\left(\mathrm{dd}, 1 \mathrm{H}, J=11.6,2.6 \mathrm{~Hz}, \mathrm{OCH}_{2}\right), 6.8(\mathrm{~d}, 2 \mathrm{H}$, $J=8.0,5,6$-Ar-H homoveratryl), 7.17 (s, 1H, 2-Ar-H homoveratryl), 7.65 (t, 2H, $J=8.5,6,7-\mathrm{Ar}-\mathrm{H}$ ), 8.14 (d, 4H, $J=7.5,3,4,5,8-A r-H), 8.62$ (s, 1H, 1-Ar-H). MS m/z (\%); 424 (12.07, $\left.\mathrm{M}^{+}+2\right), 423$ (9.11, $\left.\mathrm{M}^{+}+1\right), 181$ (11.91), $152(100), 137$ (28.19).

1-(Biphenyl-4-yl)ethanone O-2-hydroxy-3-(isopropylamino)propyl oxime, hydrogen oxalate (4m). Yield 60\%; M.p. $169-171{ }^{\circ} \mathrm{C}$; ${ }^{1} \mathrm{H}-\mathrm{NMR}$ (free base in DMSO- $\left.d_{6}\right) \delta 1.21\left(\mathrm{~d}, 6 \mathrm{H}, J=6.5,2 \mathrm{CH}_{3}\right.$ ), 2.16 (s, $\left.3 \mathrm{H}, \mathrm{CH}_{3} \mathrm{C}\right), 2.95-3.12\left(\mathrm{~m}, 3 \mathrm{H}, \mathrm{CH}_{2} \mathrm{~N}, \mathrm{C} \underline{\mathrm{H}}\left(\mathrm{CH}_{3}\right)\right), 3.45-3.72(\mathrm{~m}, 3 \mathrm{H}, \mathrm{C} \underline{\mathrm{HOH}}, \mathrm{OH}$ and $\mathrm{NH}$ exchangeable with $\left.\mathrm{D}_{2} \mathrm{O}\right), 3.89\left(\mathrm{dd}, 1 \mathrm{H}, J=10.9,6.1 \mathrm{~Hz}, \mathrm{OCH}_{2}\right), 4.29\left(\mathrm{dd}, 1 \mathrm{H}, J=11.0,2.5 \mathrm{~Hz}, \mathrm{OCH}_{2}\right), 7.2-7.4$ (m, 3H, 3', '4', 5'-Ar-H), 7.56 (d, 2H, $\left.J=8.0,2^{\prime}, 6^{\prime}-\mathrm{Ar}-\mathrm{H}\right), 7.84$ (d, 4H, $\left.J=8.5,2,3,5,6-\mathrm{Ar}-\mathrm{H}\right)$.

1-(Biphenyl-4-yl)ethanone O-3-(tert-butylamino)-2-hydroxypropyl oxime, hydrochloride (4n). Yield 52\%; M.p. $165-167{ }^{\circ} \mathrm{C} ;{ }^{1} \mathrm{H}$ NMR (free base in DMSO- $\left.d_{6}\right) \delta 1.21\left(\mathrm{~s}, 9 \mathrm{H}, 3 \mathrm{CH}_{3}\right), 2.16\left(\mathrm{~s}, 3 \mathrm{H}, \mathrm{CH}_{3} \mathrm{C}\right.$ ), 2.95-3.12 (m, 3H, $\left.\mathrm{CH}_{2} \mathrm{~N}, \mathrm{C} \underline{\mathrm{H}}\left(\mathrm{CH}_{3}\right)\right), 3.45-3.72(\mathrm{~m}, 3 \mathrm{H}, \mathrm{C} \underline{\mathrm{HOH}}, \mathrm{OH}$ and $\mathrm{NH}$ exchangeable with $\left.\mathrm{D}_{2} \mathrm{O}\right), 3.89\left(\mathrm{dd}, 1 \mathrm{H}, J=10.9,6.1 \mathrm{~Hz}, \mathrm{OCH}_{2}\right), 4.29\left(\mathrm{dd}, 1 \mathrm{H}, J=11.0,2.5 \mathrm{~Hz}, \mathrm{OCH}_{2}\right), 7.2-7.4(\mathrm{~m}$, $\left.3 \mathrm{H}, 3^{\prime}, 4^{\prime}, 5^{\prime}-\mathrm{Ar}-\mathrm{H}\right), 7.56$ (d, 2H, $\left.J=8.0,2^{\prime}, 6^{\prime}-\mathrm{Ar}-\mathrm{H}\right), 7.84(\mathrm{~d}, 4 \mathrm{H}, J=8.5,2,3,5,6-\mathrm{Ar}-\mathrm{H}) . \mathrm{MS} m / z$ (\%); $342\left(8.39, \mathrm{M}^{+}+2\right), 341$ (7.71, $\left.\mathrm{M}^{+}+1\right), 193$ (69), 153 (39.43), 86 (100).

1-(Biphenyl-4-yl)ethanone O-3-(3,4-dimethoxyphenethylamino)-2-hydroxypropyl oxime, hydrochloride (4o). Yield 48\%; M.p. 157-159 ${ }^{\circ} \mathrm{C}$; ${ }^{1} \mathrm{H}-\mathrm{NMR}$ (free base in $\mathrm{CDCl}_{3}$ ) $\delta 2.2\left(\mathrm{~s}, 3 \mathrm{H}, \mathrm{CH}_{3}\right), 2.85-3.1$ (m, $\left.6 \mathrm{H}, \underline{\mathrm{C}}_{2} \mathrm{NHC}_{2} \mathrm{CH}_{2}\right), 3.45-3.72\left(\mathrm{~m}, 3 \mathrm{H}, \mathrm{C} \underline{\mathrm{HOH}}, \mathrm{OH}\right.$ and $\mathrm{NH}$ exchangeable with $\mathrm{D}_{2} \mathrm{O}$ ), 3.85 (s, $\left.6 \mathrm{H}, 2 \mathrm{OCH}_{3}\right) 4.02\left(\mathrm{dd}, 1 \mathrm{H}, J=11.2,6.2 \mathrm{~Hz}, \mathrm{OCH}_{2}\right), 4.22\left(\mathrm{dd}, 1 \mathrm{H}, J=11.5,2.2 \mathrm{~Hz}, \mathrm{OCH}_{2}\right), 6.85$ (d, 2H, $J=8.0,5,6$-Ar-H homoveratryl), 7.25 (s, 1H, 2-Ar-H homoveratryl), 7.3-7.5 (m, 3H, 3', $\left.4^{\prime}, 5^{\prime}-\mathrm{Ar}-\mathrm{H}\right), 7.59$ (d, 2H, $\left.J=8.0,2^{\prime}, 6^{\prime}-\mathrm{Ar}-\mathrm{H}\right), 7.88$ (d, 4H, $\left.J=8.5,2,3,5,6-\mathrm{Ar}-\mathrm{H}\right) . \mathrm{MS} m / z(\%)$; $450\left(11.9, \mathrm{M}^{+}+2\right), 449$ (6.12, $\left.\mathrm{M}^{+}+1\right), 297$ (34.08), 194 (100).

1-(5-Chlorothiophen-2-yl)ethanone O-2-hydroxy-3-(isopropyl amino)propyl oxime, hydrochloride (4p). Yield 70\%; M.p. $145-147{ }^{\circ} \mathrm{C}$; ${ }^{1} \mathrm{H}-\mathrm{NMR}$ (free base in DMSO- $\left.d_{6}\right) \delta 1.03\left(\mathrm{~d}, 6 \mathrm{H}, J=7.0,2 \mathrm{CH}_{3}\right), 2.10(\mathrm{~s}, 3 \mathrm{H}$, $\left.\left.\mathrm{CH}_{3} \mathrm{C}=\mathrm{NO}\right), 2.85-3.06\left(\mathrm{~m}, 3 \mathrm{H}, \mathrm{CH}_{2} \mathrm{~N}, \mathrm{C} \underline{\mathrm{H}}\left(\mathrm{CH}_{3}\right)_{2}\right)\right), 3.5-3.7$ (m, 3H, $\mathrm{C} \underline{\mathrm{HOH}}, \mathrm{OH}$ and $\mathrm{NH}$ exchangeable 
with $\left.\mathrm{D}_{2} \mathrm{O}\right), 4.11\left(\mathrm{dd}, 1 \mathrm{H}, J=11.5,6.2 \mathrm{~Hz}, \mathrm{OCH}_{2}\right), 4.39\left(\mathrm{dd}, 1 \mathrm{H}, J=11.5,2.2 \mathrm{~Hz}, \mathrm{OCH}_{2}\right), 7.17(\mathrm{~d}, 1 \mathrm{H}$, $J=4.0,3$-thiophene-H), 7.35 (d, 1H, $J=4.1$, 4-thiophene -H).

1-(5-Chlorothiophen-2-yl)ethanone O-3-(tert-butylamino)-2-hydroxypropyl oxime, hydrochloride (4q). Yield 62\%; M.p. $152-154{ }^{\circ} \mathrm{C} ;{ }^{1} \mathrm{H}-\mathrm{NMR}$ (free base in DMSO-d $d_{6} \delta 1.33\left(\mathrm{~s}, 9 \mathrm{H}, 3 \mathrm{CH}_{3}\right), 2.21(\mathrm{~s}, 3 \mathrm{H}$, $\left.\mathrm{CH}_{3} \mathrm{C}=\mathrm{NO}\right), 2.77-2.96\left(\mathrm{~m}, 2 \mathrm{H}, \mathrm{CH}_{2} \mathrm{~N}\right) 3.47-3.66(\mathrm{~m}, 3 \mathrm{H}, \mathrm{CHOH}, \mathrm{OH}$ and $\mathrm{NH}$ exchangeable with $\left.\mathrm{D}_{2} \mathrm{O}\right), 4.08\left(\mathrm{dd}, 1 \mathrm{H}, J=11.4,6.5 \mathrm{~Hz}, \mathrm{OCH}_{2}\right), 4.38\left(\mathrm{dd}, 1 \mathrm{H}, J=11.3,2.8 \mathrm{~Hz}, \mathrm{OCH}_{2}\right), 6.98(\mathrm{~d}, 1 \mathrm{H}$, $J=4.2$, 3-thiophene-H), 7.37 (d, $1 \mathrm{H}, J=4.0$, 4-thiophene-H). MS $m / z(\%) ; 306\left(2.06, \mathrm{M}^{+}+2\right), 305$ (1.45, $\left.\mathrm{M}^{+}+1\right), 160$ (100), 145 (82.20), 72 (84.98).

1-(5-Chlorothiophen-2-yl)ethanone O-3-(3,4-dimethoxyphenethylamino)-2-hydroxypropyl oxime, hydrochloride (4r). Yield 52\%; M.p. $148-150{ }^{\circ} \mathrm{C}$; ${ }^{1} \mathrm{H}-\mathrm{NMR}$ (free base in DMSO- $d_{6}$ ) $\delta 2.19$ (s, 3H, $\mathrm{CH}_{3} \mathrm{C}=\mathrm{NO}$ ), 2.79-3.05 (m, 6H, $\left.\underline{\mathrm{C}}_{2} \mathrm{NHC}_{2} \mathrm{CH}_{2}\right)$, 3.4-3.6 (m, 3H, $\underline{\mathrm{CHOH}}, \mathrm{OH}$ and $\mathrm{NH}$ exchangeable with $\left.\mathrm{D}_{2} \mathrm{O}\right), 4.0\left(\mathrm{dd}, 1 \mathrm{H}, J=11.0,6.0 \mathrm{~Hz}, \mathrm{OCH}_{2}\right), 4.32\left(\mathrm{dd}, 1 \mathrm{H}, J=11.5,2.6 \mathrm{~Hz}, \mathrm{OCH}_{2}\right), 6.88(\mathrm{~d}, 2 \mathrm{H}$, $J=8.0,5,6$-Ar-H homoveratryl), 7.17 (d, 1H, $J=4.0$, 3-thiophene-H), 7.29 (s, 1H, 2-Ar-H homoveratryl), 7.39 (d, 1H, $J=4.01$, 4-thiophene -H). MS m/z (\%); 413 (3.22, $\left.\mathrm{M}^{+}\right), 261$ (77.44), 158 (100), 73 (13.79).

1,3,3-Trimethylbicyclo[2.2.1] heptan-2-one O-2-hydroxy-3-(isopropylamino)propyl oxime, hydrochloride (4s). Yield 55\%; M.p. $127-129{ }^{\circ} \mathrm{C}$; ${ }^{1} \mathrm{H}-\mathrm{NMR}$ (free base in $\left.\mathrm{CDCl}_{3}\right) \delta 1.14\left(\mathrm{~d}, 6 \mathrm{H}, J=6.5,2 \mathrm{CH}_{3}\right.$ ), 1.25 (s, $\left.6 \mathrm{H}, 2 \mathrm{CH}_{3}\right), 1.32\left(\mathrm{~s}, 3 \mathrm{H}, \mathrm{CH}_{3}\right), 1.44-1.81\left(\mathrm{~m}, 7 \mathrm{H}, \mathrm{CH}, 3 \mathrm{CH}_{2}\right.$ cyclic moiety), 2.7-3.0 (m, $\left.2 \mathrm{H}, \mathrm{CH}_{2} \mathrm{~N}\right)$, 3.33-3.71 (m, 3H, $\underline{\mathrm{HOH}}, \mathrm{OH}$ and $\mathrm{NH}$ exchangeable with $\left.\mathrm{D}_{2} \mathrm{O}\right), 4.11\left(\mathrm{dd}, 1 \mathrm{H}, J=11.2,6.5 \mathrm{~Hz}, \mathrm{OCH}_{2}\right)$, 4.31 (dd, 1H, $J=11.7,2.5 \mathrm{~Hz}, \mathrm{OCH}_{2}$ ). MS m/z (\%); 284 (11.25, $\left.\mathrm{M}^{+}+2\right), 167$ (12.71), 81 (100), 69 (36.25).

1,3,3-Trimethylbicyclo[2.2.1] heptan-2-one O-3-(tert-butylamino)-2-hydroxypropyl oxime, hydrogen oxalate (4t). Yield 52\%; M.p. $115-117{ }^{\circ} \mathrm{C} ;{ }^{1} \mathrm{H}-\mathrm{NMR}$ (free base in $\mathrm{CDCl}_{3}$ ) $\delta 1.12\left(\mathrm{~s}, 9 \mathrm{H}, 3 \mathrm{CH}_{3}\right), 1.25$ $\left(\mathrm{s}, 6 \mathrm{H}, 2 \mathrm{CH}_{3}\right), 1.34\left(\mathrm{~s}, 3 \mathrm{H}, \mathrm{CH}_{3}\right), 1.4-1.8\left(\mathrm{~m}, 7 \mathrm{H}, \mathrm{CH}, 3 \mathrm{CH}_{2}\right.$ cyclic moiety), 2.9-3.4 (m, $\left.2 \mathrm{H}, \mathrm{CH}_{2} \mathrm{~N}\right)$, 3.65-3.9 (m, 3H, $\mathrm{CHOH}, \mathrm{OH}$ and $\mathrm{NH}$ exchangeable with $\left.\mathrm{D}_{2} \mathrm{O}\right), 3.88(\mathrm{dd}, 1 \mathrm{H}, J=11.5,6.5 \mathrm{~Hz}$, $\left.\mathrm{OCH}_{2}\right), 4.21\left(\mathrm{dd}, 1 \mathrm{H}, J=11.0,2.8 \mathrm{~Hz}, \mathrm{OCH}_{2}\right)$.

1,3,3-Trimethylbicyclo[2.2.1] heptan-2-one O-3-(3,4-dimethoxyphenethylamino)-2-hydroxypropyl oxime, hydrogen oxalate (4u). Yield 39\%; M.p. $140-142{ }^{\circ} \mathrm{C}$; ${ }^{1} \mathrm{H}-\mathrm{NMR}$ (free base in DMSO- $\left.d_{6}\right) \delta 1.22(\mathrm{~s}, 6 \mathrm{H}$, $\left.2 \mathrm{CH}_{3}\right), 1.31\left(\mathrm{~s}, 3 \mathrm{H}, \mathrm{CH}_{3}\right), 1.5-1.78\left(\mathrm{~m}, 7 \mathrm{H}, \mathrm{CH}, 3 \mathrm{CH}_{2}\right.$ in cyclic moiety), 2.4-2.9 (m, 6H, $\left.\underline{\mathrm{C}}_{2} \mathrm{NHC}_{2} \underline{\mathrm{C}}_{2}\right), 3.61-3.81\left(\mathrm{~m}, 3 \mathrm{H}, \mathrm{C} \underline{\mathrm{HOH}}, \mathrm{OH}\right.$ and $\mathrm{NH}$ exchangeable with $\left.\mathrm{D}_{2} \mathrm{O}\right), 3.97$ (s, 6H, $\left.\mathrm{OCH}_{3}\right), 4.10\left(\mathrm{dd}, 1 \mathrm{H}, J=11.6,6.4 \mathrm{~Hz}, \mathrm{OCH}_{2}\right), 4.22\left(\mathrm{dd}, 1 \mathrm{H}, J=11.4,2.5 \mathrm{~Hz}, \mathrm{OCH}_{2}\right), 6.9(\mathrm{~d}, 2 \mathrm{H}$, $J=8.5,5,6-\mathrm{Ar}-\mathrm{H}), 7.15$ (s, 1H, 2-Ar-H). MS m/z (\%); 406 (9.17, $\left.\mathrm{M}^{+}+2\right), 405\left(7.45, \mathrm{M}^{+}+1\right), 253$ (26.99), 151 (50.87), 104 (88.50), 81 (100).

5-Methoxy-3,4-dihydronaphthalen-1(2H)-one O-2-hydroxy-3-(isopropylamino)propyl oxime, hydrogen oxalate (4v). Yield 76\%; M.p. ${ }^{180-182 ~}{ }^{\circ} \mathrm{C} ;{ }^{1} \mathrm{H}-\mathrm{NMR}$ (free base in $\left.\mathrm{CDCl}_{3}\right) \delta 1.21(\mathrm{~d}, 6 \mathrm{H}, J=6.5$, $\left.2 \mathrm{CH}_{3}\right), 2.22-2.61\left(\mathrm{~m}, 6 \mathrm{H}, 3 \mathrm{CH}_{2}\right.$ cyclic structure), 2.8-3.1 (m, 3H, $\left.\mathrm{CH}_{2} \mathrm{NCH}\right), 3.5-3.7(\mathrm{~m}, 3 \mathrm{H}$,

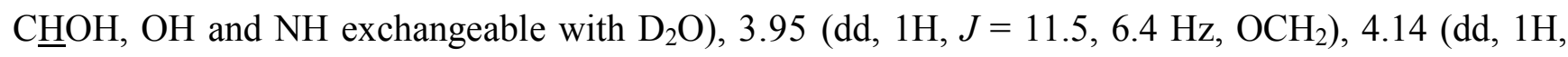
$\left.J=11.6,2.6 \mathrm{~Hz}, \mathrm{OCH}_{2}\right), 4.19\left(\mathrm{~s}, 3 \mathrm{H}, \mathrm{OCH}_{3}\right), 7.11(\mathrm{~d}, 1 \mathrm{H}, J=9.0,6-\mathrm{Ar}-\mathrm{H}), 7.36(\mathrm{t}, 1 \mathrm{H}, J=8.5,7-\mathrm{Ar}-\mathrm{H})$, 7.55 (d, 1H, $J=8.5,8$-Ar-H). MS $m / z(\%) ; 308$ (6.55, $\left.\mathrm{M}^{+}+2\right), 307$ (3.45, $\left.\mathrm{M}^{+}+2\right), 248$ (35), 174 (100). 
5-Methoxy-3,4-dihydronaphthalen-1(2H)-one O-3-(tert-butylamino)-2-hydroxypropyl oxime, hydrogen oxalate (4w). Yield 69\%; M.p. $177-179{ }^{\circ} \mathrm{C} ;{ }^{1} \mathrm{H}-\mathrm{NMR}$ (free base in $\left.\mathrm{CDCl}_{3}\right) \delta 1.21\left(\mathrm{~s}, 9 \mathrm{H}, 3 \mathrm{CH}_{3}\right.$ ), 2.03-2.81 (m, $6 \mathrm{H}, 3 \mathrm{CH}_{2}$ cyclic structure), 3.1-3.4 (m, $\left.2 \mathrm{H}, \mathrm{CH}_{2} \mathrm{~N}\right), 3.5-3.7(\mathrm{~m}, 3 \mathrm{H}, \mathrm{CHOH}, \mathrm{OH}$ and $\mathrm{NH}$ exchangeable with $\left.\mathrm{D}_{2} \mathrm{O}\right), 3.89\left(\mathrm{dd}, 1 \mathrm{H}, J=11.5,6.2 \mathrm{~Hz}, \mathrm{OCH}_{2}\right), 4.08(\mathrm{dd}, 1 \mathrm{H}, J=11.6,2.4 \mathrm{~Hz}$, $\left.\mathrm{OCH}_{2}\right), 4.15\left(\mathrm{~s}, 3 \mathrm{H}, \mathrm{OCH}_{3}\right), 7.07(\mathrm{~d}, 1 \mathrm{H}, J=9.0,6-\mathrm{Ar}-\mathrm{H}), 7.31(\mathrm{t}, 1 \mathrm{H}, J=8.5,7-\mathrm{Ar}-\mathrm{H}), 7.52(\mathrm{~d}, 1 \mathrm{H}$, $J=8.5,8-$ Ar-H).

5-Methoxy-3,4-dihydronaphthalen-1(2H)-one O-3-(3,4-dimethoxyphenethylamino)-2-hydroxypropyl oxime, hydrogen oxalate (4x). Yield 58\%; M.p. $160-162{ }^{\circ} \mathrm{C} ;{ }^{1} \mathrm{H}-\mathrm{NMR}$ (free base in DMSO- $d_{6}$ ) $\delta$ 2.1-2.9 (m, 12H, $\underline{\mathrm{C}}_{2} \mathrm{NHC}_{2} \mathrm{NH}_{2}, 3 \mathrm{CH}_{2}$ cyclic structure), 3.51-3.70 (m, 3H, $\mathrm{C} \underline{\mathrm{HOH}}, \mathrm{OH}$ and $\mathrm{NH}$ exchangeable with $\left.\mathrm{D}_{2} \mathrm{O}\right), 3.82\left(\mathrm{~s}, 6 \mathrm{H}, 2 \mathrm{OCH}_{3}\right), 3.88\left(\mathrm{~s}, 3 \mathrm{H}, \mathrm{OCH}_{3}\right), 4.11(\mathrm{dd}, 1 \mathrm{H}, J=11.1,6.4 \mathrm{~Hz}$, $\mathrm{OCH}_{2}$ ), 4.27 (dd, $\left.1 \mathrm{H}, J=11.3,3.0 \mathrm{~Hz}, \mathrm{OCH}_{2}\right), 6.79$ (d, 2H, $J=8.5,5,6$-Ar-H homoveratryl), 7.0 (s, $1 \mathrm{H}, 2$-Ar-H homoveratryl), 7.25 (d, 1H, $J=8.0,6$-Ar-H), 7.47 (t, 1H, $J=9.0,7-A r-H), 7.62$ (d, 1H, $J=8.0,8-\mathrm{Ar}-\mathrm{H})$.

Diphenylmethanone O-3-(3,4-dimethoxyphenethylamino)-2-hydroxypropyl oxime, hydrochloride (4y). Yield 52\%; M.p. $166-168{ }^{\circ} \mathrm{C} ;{ }^{1} \mathrm{H}-\mathrm{NMR}$ (free base in DMSO- $\left.d_{6}\right) \delta 2.58-2.96\left(\mathrm{~m}, 6 \mathrm{H}, \mathrm{C}_{2} \mathrm{NHC}_{2} \underline{\mathrm{C}}_{2} \underline{\mathrm{H}}_{2}\right.$ ), 3.6-3.9 (m, 3H, CㅍH, $\mathrm{OH}$ and $\mathrm{NH}$ exchangeable with $\left.\mathrm{D}_{2} \mathrm{O}\right), 3.95\left(\mathrm{~s}, 6 \mathrm{H}, 2 \mathrm{OCH}_{3}\right), 4.16(\mathrm{dd}, 1 \mathrm{H}$, $\left.J=10.8,5.9 \mathrm{~Hz}, \mathrm{OCH}_{2}\right), 4.28\left(\mathrm{dd}, 1 \mathrm{H}, J=11.4,3.4 \mathrm{~Hz}, \mathrm{OCH}_{2}\right), 6.67(\mathrm{~d}, 2 \mathrm{H}, J=8.5,5,6-\mathrm{Ar}-\mathrm{H}$ homoveratryl), 7.18 (s, 1H, 2-Ar-H homoveratryl), 7.60-8.02 (m, 10H, Ar-H). MS m/z (\%); 436 (5.85, $\left.\mathrm{M}^{+}+2\right), 435$ (6.79, $\left.\mathrm{M}^{+}+1\right), 152$ (100), 137 (26.50).

1-Phenylethan-1-one O-3-(3,4-dimethoxyphenethylamino)-2-hydroxypropyl oxime, hydrochloride (4z). Yield 56\%; M.p. $138-141{ }^{\circ} \mathrm{C}$; ${ }^{1} \mathrm{H}-\mathrm{NMR}$ (free base in DMSO- $\left.d_{6}\right) \delta 1.91\left(\mathrm{~s}, 3 \mathrm{H}, \mathrm{CH}_{3}\right), 2.4-2.9(\mathrm{~m}, 6 \mathrm{H}$, $\left.\mathrm{C}_{2}{ }_{2} \mathrm{NHCH}_{2} \underline{\mathrm{C}}_{2}\right), 3.6-3.9$ (m, 3H, $\underline{\mathrm{HOH}}, \mathrm{OH}$ and $\mathrm{NH}$ exchangeable with $\left.\mathrm{D}_{2} \mathrm{O}\right), 3.90\left(\mathrm{~s}, 6 \mathrm{H}, 2 \mathrm{OCH}_{3}\right)$, $4.05\left(\mathrm{dd}, 1 \mathrm{H}, J=11.2,6.4 \mathrm{~Hz}, \mathrm{OCH}_{2}\right), 4.25\left(\mathrm{dd}, 1 \mathrm{H}, J=11.9,2.7 \mathrm{~Hz}, \mathrm{OCH}_{2}\right), 6.87$ (d, 2H, 5,6-Ar-H homoveratryl), 7.15 (s, 1H, 2-Ar-H homoveratryl), 7.53 (t, 3H, $J=8, \operatorname{Ar}-\mathrm{H}), 7.9$ (d, 2H, $J=8.5$, Ar-H). MS $m / z(\%) ; 374$ (5.97, $\left.\mathrm{M}^{+}+2\right), 375$ (7.45, $\left.\mathrm{M}^{+}+1\right), 165$ (19.97), 118 (100), 77 (29.65).

\subsection{Molecular Docking Methodology}

Automated docking simulations were conducted with the Molegro Virtual Docker 2007 (MVD 2007.2.2.5-Aug 27, 2007 [win32]) fully functional free trial version with time limiting license [20] 3D Molecular structures and energy minimization were carried out by free version of Marvinsketch 4.1.13 from Chemaxon Ltd. (Budapest, Hungary) [28]. 3D crystal structures of $\beta_{1}$ and $\beta_{2}$-adrenergic receptors were obtained from Protein Data Bank (PDB); entries 2VT4 and 2R4R, respectively [18,19,29]. All the molecular modeling studies were carried out on a PC equipped with an Intel Celeron $1.2 \mathrm{GHz}$ processor, and $320 \mathrm{MB}$ memory running the Windows XP operating system.

The docking study was performed, in the present investigation, following a general procedure for docking [30,31]. The coordinates of the crystal structures adrenergic receptors were imported into Molegro Virtual Docker. Receptor structures were checked for missing atoms, bonds and contacts. The ligand molecules were constructed in 2D form then converted to 3D form and energy minimized by Marvinsketch. Energy minimized conformers were imported into Molegro Virtual Docker. The 
dimensions of the docking constraint were manipulated so as to accommodate all the amino acid residues present in the active site (radius $12 \AA$ ). The ligands were manually docked into the binding cavity of $\beta_{2}$ adrenergic receptor, guided by all the amino acids present in the active site, and snapshots were recorded. Figure 2a,b represent the docking snapshots of compounds $\mathbf{4 o}$ and $\mathbf{4 q}$ with the $\beta_{1}$ and $\beta_{2}$-adrenergic receptors, respectively, showing the forces of interaction. In this study the MolDock score function and hydrogen bond interactions between tested compounds and the target receptors were used to compare between the tested compounds and reference drugs. The MolDock score is a new technique for high-accuracy molecular docking. It is an extension of the piecewise linear potential (PLP) [32,33] including new hydrogen bonding and electrostatic terms. To further improve docking accuracy, a re-ranking scoring function is introduced, which identifies the most promising docking solution from the solutions obtained by the docking algorithm [34]. The lower MolDock score means better ligand-receptor interactions. The MolDock scores and hydrogen bond were recorded (Table 3).

\subsection{Biological Evaluation}

Epinephrine hydrochloride injection $(0.25 \mathrm{mg} / 1 \mathrm{~mL})$ was obtained from Misr Co. Cairo, Egypt. Propranolol hydrochloride (Inderal injection) B.P. 0.1\%, was obtained from AstraZeneca Limited (Cairo, Egypt). Acetylcholine chloride and salbutamol sulfate were purchased from Sigma-Aldrich chemical Co. All other chemicals used in this study were of finest analytical grade, and obtained from El-Nasr Pharmaceutical Chemicals Company. Tested compounds were dissolved in distilled water or $20 \%$ ethanol, just before use, in $10^{-5}$ mole concentration. Adult male Hartley guinea pigs weighing 350-500 g were used. Animals were maintained under standard conditions of temperature with regular $12 \mathrm{~h} \mathrm{light/dark}$ cycles and allowed free access to standard laboratory food and water. Biological evaluation using animals was performed in accordance with the Ethics Committee of faculty of Pharmacy Mansoura University.

\subsubsection{In Vitro Screening for $\beta_{1}$-Adrenoceptor Activity in Isolated Guinea Pig Atria}

Guinea pigs were anaesthetized by diethyl ether. The chest was opened and the heart was quickly excised and placed in oxygenated Ringer Locke solution of the following composition $(\mathrm{g} / \mathrm{L}): \mathrm{NaCl}(9)$, $\mathrm{KCl}(0.42), \mathrm{CaCl}_{2}(0.24), \mathrm{NaHCO}_{3}(0.15)$ and glucose (1). Atria were dissected away from the rest of the heart, freed from connective tissue and suspended in $50 \mathrm{~mL}$ organ bath containing Ringer Locke solution. This solution is thermoregulated at $37{ }^{\circ} \mathrm{C}$ and continuously bubbled with pure oxygen. The extremities of the strip, consisting of both atria, were tied with a thread. The first thread was used to tie the organ to a muscle holder fixed in place in a muscle chamber of an isolate organ bath; the second thread was used to connect atria to an isomeric force-displacement transducer (model 50-7905, Harvard Apparatus Inc, South Natick, MA, USA) situated above the muscle chamber and connected to a twochannel oscillograph (model 50-8622, Harvard Apparatus). Atria spontaneously beating were loaded with $0.5 \mathrm{~g}$ and were allowed to adjust to the bath conditions for at least $30 \mathrm{~min}$ prior to the experiment, and the Ringer Locke solution was changed at 10 min intervals. Epinephrine ( $43 \mu \mathrm{L}$ of epinephrine vial; $10^{-6} \mathrm{~mol}$ ) was applied and allowed to act till the response was fully developed. The atria were washed with Ringer Locke solution. Test compound $\left(10^{-5} \mathrm{~mol}, 2.5-4.5 \mathrm{mg} / \mathrm{L}\right)$ was applied and incubated for $5 \mathrm{~min}$ in the organ bath to test and evaluate any possible agonistic activity on the $\beta_{1}$-adrenergic receptor. 
Then, epinephrine (43 $\mu \mathrm{L}$ of epinephrine vial; $10^{-6} \mathrm{~mol}$ ) was added in the organ bath in order to evaluate any competitive antagonistic activity of the test compound to epinephrine on the $\beta_{1}$-adrenergic receptor. Propranolol $\left(10^{-5} \mathrm{~mol}\right)$ was used as reference drug.

\subsubsection{In Vitro Screening for $\beta_{2}$-Adrenoceptor Activity in Isolated Guinea Pig Trachea}

Guinea pig was anaesthetized by diethylether; the trachea was carefully isolated and immersed in oxygenated Krebs-Hanseleit solution of the following composition $(\mathrm{g} / \mathrm{L}): \mathrm{NaCl}(6.9), \mathrm{KCl}(0.35)$, $\mathrm{KH}_{2} \mathrm{PO}_{4}(0.16), \mathrm{NaHCO}_{3}(2.1), \mathrm{MgSO}_{4}(0.29)$, anhydrous $\mathrm{CaCl}_{2}$ (0.28) and glucose (2). The tracheal preparation was cautiously cleaned of unnecessary adipose and connective tissues. Subsequently, the tracheal cartilage containing smooth muscles was cut into zig-zag strips according to Emmerson and Mackay method [35]. Zig-zag tracheal strips were tied at each end with a thread. The first thread was used to tie the organ to a muscle holder fixed in place in a muscle chamber of an isolate organ bath; the second thread was used to connect trachea to an isomeric force-displacement transducer (model 50-7905, Harvard Apparatus) situated above the muscle chamber and connected to a two-channel oscillograph (model 50-8622, Harvard Apparatus). The organ bath contains $50 \mathrm{~mL}$ of Krebs-Hanseleit solution at $32{ }^{\circ} \mathrm{C}$ and gassed with pure oxygen. The zig-zag tracheal strips were loaded with $1 \mathrm{~g}$ and were left to stabilize for $1 \mathrm{~h}$, and the Krebs-Hanseleit solution was changed at $15 \mathrm{~min}$ intervals. The zig-zag tracheal strips were precontracted with acetylcholine chloride $\left(0.9 \mathrm{mg} / 50 \mathrm{~mL} ; 10^{-4} \mathrm{~mol}\right)$ and allowed to act till the response was fully developed. The test compound $\left(10^{-5} \mathrm{~mol}\right)$ was then added and incubated for $20 \mathrm{~min}$ in the organ bath to evaluate its agonistic activity on $\beta_{2}$-adrenergic receptor. Salbutamol sulfate $\left(10^{-5} \mathrm{~mol}\right)$ was then added in organ bath in the presence of the test compound in order to evaluate any blocking activity of the test compound to $\beta_{2^{-}}$adrenergic receptor. Propranolol $\left(10^{-5} \mathrm{~mol}\right)$ was used as reference drug.

\section{Conclusions}

In this study, a series of oxime ether derivatives $\mathbf{4 a}-\mathbf{z}$ were designed and successfully synthesized in convenient steps. Selected compounds were tested for their biological activities against in vitro $\beta_{1}$ - and $\beta_{2}$-adrenergic receptors and also examine their binding energies with $3 \mathrm{D}$ crystal structures of $\beta_{1}$ and $\beta_{2}$ receptors by doing molecular docking analysis. These findings confirm the significance of a homoveratrylamine portion for $\beta_{1}$ receptors and also indicate the importance of the presence of a chlorothiophene moiety in the hydrophobic region for best complementarity with $\beta_{2}$ receptors.

\section{Supplementary Materials}

Supplementary materials can be accessed at: http://www.mdpi.com/1420-3049/19/3/3417/s1.

\section{Acknowledgments}

Authors would like to thank Ghada M. Suddek, Associate Professor of Pharmacology, Faculty of Pharmacy, Mansoura University for performing the biological screening. HAG thanks the Deanship of Scientific Research and the Research Center, College of Pharmacy, King Saud University. 


\section{Conflicts of Interest}

Authors have no conflict of interest to declare in connection with the contents of this manuscript.

\section{References}

1. Hoffman, B.B. Adrenoceptor antagonist drugs. In Basic and Clinical Pharmacology, 10th ed.; Katzung, B.G., Ed.; McGraw-Hill: New York, NY, USA, 2007.

2. Imbs, J.L.; Miesch, F.; Schwartz, J.; Velly, J.; Leclerc, G.; Mann, A.; Wermuth, C.G. A potent new beta2-adrenoceptor blocking agent. Br. J. Pharmacol. 1977, 60, 357-362.

3. Leclerc, G.; Mann, A.; Wermuth, C.G.; Bieth, N.; Schwartz, J. Synthesis and beta-adrenergic blocking activity of a novel class of aromatic oxime ethers. J. Med. Chem. 1977, 20, 1657-1662.

4. Bouzoubaa, M.; Leclerc, G.; Decker, N.; Schwartz, J.; Andermann, G. Synthesis and beta-adrenergic blocking activity of new aliphatic and alicyclic oxime ethers. J. Med. Chem. 1984, 27, 1291-1294.

5. Hothersall, J.D.; Black, J.; Caddick, S.; Vinter, J.G.; Tinker, A.; Baker, J.R. The design, synthesis and pharmacological characterization of novel beta(2)-adrenoceptor antagonists. Br. J. Pharmacol. 2011, 164, 317-331.

6. Wenzel, D.; Knies, R.; Matthey, M.; Klein, A.M.; Welschoff, J.; Stolle, V.; Sasse, P.; Roll, W.; Breuer, J.; Fleischmann, B.K. Beta(2)-adrenoceptor antagonist ici 118,551 decreases pulmonary vascular tone in mice via a $\mathrm{G}(\mathrm{i} / \mathrm{o})$ protein/nitric oxide-coupled pathway. Hypertension 2009, 54, 157-163.

7. Citterio, A.; Filippini, L. Homolytic c-alkylation of aldoximes. Synthesis 1986, 1986, 473-474.

8. Zaitsev, A.B.; Vasil'tsov, A.M.; Schmidt, E.Y.; Mikhaleva, A.I.; Morozova, L.V.; Afonin, A.V.; Ushakov, I.A.; Trofimov, B.A. $O$-vinyldiaryl-and $O$-vinylaryl (hetaryl) ketoximes: A breakthrough in $O$-vinyloxime chemistry. Tetrahedron 2002, 58, 10043-10046.

9. Maheswara, M.; Siddaiah, V.; Gopalaiah, K.; Rao, V.M.; Rao, C.V. A simple and effective glycine-catalysed procedure for the preparation of oximes. J. Chem. Res. 2006, 2006, 362-363.

10. Campbell, K.N.; Campbell, B.K.; Chaput, E.P. The reaction of grignard reagents with oximes. Ii. The action of aryl grignard reagents with mixed ketoximes. J. Org. Chem. 1943, 8, 99-102.

11. Crovetti, A.J.; Stein, R.G. Certain o-substituted thiophene oxime carbamates used as antibacterial and antifungal agents. US Patent 4,061,764, December 1977.

12. Cottingham, R. The beckmann rearrangement of fenchone oxime. J. Org. Chem. 1960, 25, 1473-1476.

13. Hardegger, E.; Redlich, D.; Gal, A. Über steroide und sexualhormone. (114. Mitteilung). Versuche zur herstellung von 4, 13-dioxychrysen-derivaten. Helv. Chim. Acta 1945, 28, 628-637.

14. Delgado, A.; Garcia, J.M.; Mauleon, D.; Minguillon, C.; Subirats, J.R.; Feliz, M.; Lopez, F.; Velasco, D. Synthesis and conformational analysis of 2-amino-1, 2, 3, 4-tetrahydro-1-naphthalenols. Can. J. Chem. 1988, 66, 517-527.

15. Balsamo, A.; Macchia, M.; Martinelli, A.; Rossello, A. The [(methyloxy) imino] methyl moiety (moimm) in the design of a new type of $\beta$-adrenergic blocking agent. Eur. J. Med. Chem. 1999, 34, 283-291. 
16. Barril, X.; Morley, S.D. Unveiling the full potential of flexible receptor docking using multiple crystallographic structures. J. Med. Chem. 2005, 48, 4432-4443.

17. Rasmussen, S.G.; Choi, H.-J.; Rosenbaum, D.M.; Kobilka, T.S.; Thian, F.S.; Edwards, P.C.; Burghammer, M.; Ratnala, V.R.; Sanishvili, R.; Fischetti, R.F. Crystal structure of the human $\beta 2$ adrenergic G-protein-coupled receptor. Nature 2007, 450, 383-387.

18. Cherezov, V.; Rosenbaum, D.M.; Hanson, M.A.; Rasmussen, S.G.; Thian, F.S.; Kobilka, T.S.; Choi, H.-J.; Kuhn, P.; Weis, W.I.; Kobilka, B.K. High-resolution crystal structure of an engineered human $\beta 2$-adrenergic g protein-coupled receptor. Science 2007, 318, 1258-1265.

19. Warne, T.; Serrano-Vega, M.J.; Baker, J.G.; Moukhametzianov, R.; Edwards, P.C.; Henderson, R.; Leslie, A.G.; Tate, C.G.; Schertler, G.F. Structure of a b 1-adrenergic g-protein-coupled receptor. Nature 2008, 454, 486-491.

20. Abdel-Aziz, H.A.; Al-Rashood, K.A.; Ghabbour, H.A.; Fun, H.K.; Chia, T.S. 1-(4-Methyl-phenyl)-2-(phenyl-sulfon-yl)ethanone. Acta Crystallogr. E 2012, 68, o1033.

21. Kitchen, D.B.; Decornez, H.; Furr, J.R.; Bajorath, J. Docking and scoring in virtual screening for drug discovery: Methods and applications. Nat. Rev. Drug Discov. 2004, 3, 935-949.

22. Tesfamariam, B.; Allen, G.T. B1-and $\beta 2$-adrenoceptor antagonist activities of ici-215001, a putative $\beta 3$-adrenoceptor agonist. Br. J. Pharmacol. 1994, 112, 55-58.

23. Akamine, E.H.; Hohman, T.C.; Nigro, D.; Carvalho, M.H.C.; de Cássia Tostes, R.; Fortes, Z.B. Minalrestat, an aldose reductase inhibitor, corrects the impaired microvascular reactivity in diabetes. J. Pharmacol. Exp. Ther. 2003, 304, 1236-1242.

24. Poli, E.; Coruzzi, G.; Bertaccini, G. Changes in the ionic environment may alter the kind of antagonism of some histamine H2-receptor blockers in the guinea pig papillary muscle. J. Pharmacol. Method. 1990, 23, 265-274.

25. Gan, L.-L.; Wang, M.-W.; Cheng, M.-S.; Pan, L. Trachea relaxing effects and beta2-selectivity of spff, a newly developed bronchodilating agent, in guinea pigs and rabbits. Biol. Pharm. Bull. 2003, 26, 323-328.

26. Hoefle, M.L.; Hastings, S.G.; Meyer, R.F.; Corey, R.M.; Holmes, A.; Stratton, C.D. Cardioselective. Beta.-adrenergic blocking agents. 1. 1-[(3, 4-dimethoxyphenethyl) amino]-3aryloxy-2-propanols. J. Med. Chem. 1975, 18, 148-152.

27. El-Ashmawy, M.; Lissavetzky, J.; Darias, V.; Martín-Herrera, D. Synthesis and pharmacological study of a thiophene analogue of moprolol and related compounds. Arch. Pharm. 1991, 324, $45-48$.

28. Abdel-Aziz, H.A.; Ghabbour, H.A.; Chantrapromma, S.; Fun, H.K. 3-Acetyl-1,5-diphenyl-1hpyrazole-4-carbonitrile. Acta Crystallogr. E 2012, 68, o1095-o1096.

29. Kassem, M.G.; Ghabbour, H.A.; Abdel-Aziz, H.A.; Fun, H.K.; Ooi, C.W. 3-Chloro-4-methylquinolin-2(1h)-one. Acta Crystallogr. E 2012, 68, o1043.

30. Pinheiro, J.R.; Bitencourt, M.; da Cunha, E.F.; Ramalho, T.C.; Freitas, M.P. Novel anti-hiv cyclotriazadisulfonamide derivatives as modeled by ligand-and receptor-based approaches. Bioorg. Med. Chem. 2008, 16, 1683-1690.

31. Sapre, N.S.; Gupta, S.; Pancholi, N.; Sapre, N. Molecular docking studies on tetrahydroimidazo[4,5,1-jk][1,4]-benzodiazepinone (TIBO) derivatives as HIV-1 NNRT inhibitors. J. Comput. Aid. Mol. Des. 2008, 22, 69-80. 
32. Gehlhaar, D.K.; Bouzida, D.; Rejto, P.A. Fully Automated and Rapid Flexible Docking of Inhibitors Covalently Bound to Serine Proteases. In Evolutionary Programming VII; Porto, V.W., Saravanan, N., Waagen, D., Eiben, A.E., Eds.; Springer: Berlin, Heidelberg, Germany, 1998; pp. 449-461.

33. Yang, J.M.; Chen, C.C. Gemdock: A generic evolutionary method for molecular docking. Proteins 2004, 55, 288-304.

34. Thomsen, R.; Christensen, M.H. Moldock: A new technique for high-accuracy molecular docking. J. Med. Chem. 2006, 49, 3315-3321.

35. Emmerson, J.; Mackay, D. The zig-zag tracheal strip. J. Pharm. Pharmacol. 1979, 31, 798-798.

Sample Availability: Samples of the compounds $\mathbf{4 y}-\mathbf{z}$ are available from the authors.

(C) 2014 by the authors; licensee MDPI, Basel, Switzerland. This article is an open access article distributed under the terms and conditions of the Creative Commons Attribution license (http://creativecommons.org/licenses/by/3.0/). 